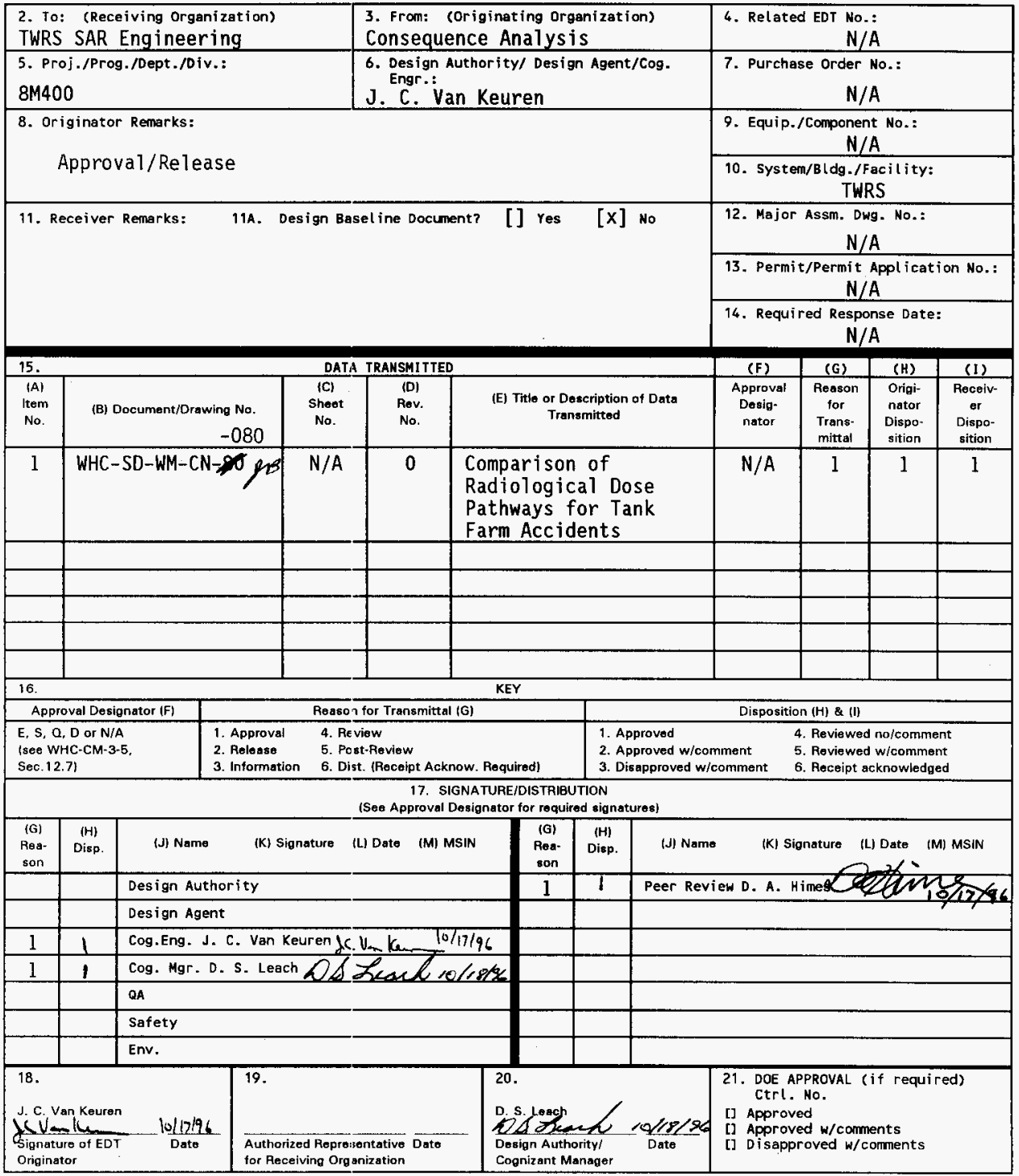

BD-7400-172-2 (05/96) GEF097 


\title{
Comparison of Radiological Dose Pathways for Tank Farm Acicidents
}

\author{
J. C. Van Keuren \\ West inghouse Hanford Co., Richland, WA 99352 \\ U.S. Department of Energy Contract DE-AC06-87RL10930 \\ EDT/ECN : EDT $619416 \quad$ UC: 510 \\ Org Code: 8M400 Charge Code: N1FW1 \\ B\&R Code: EW312007l Total Pages: 65
}

Key Words: FSAR, Radiological Consequence, $X / Q$

Abstract: This calculation note documents an evaluation of the doses from submersion and ground shine due to a release of tank farm radioactive materials, and a comparison of these doses to the doses from inhalation of the materials. The submersion and ground shine doses are insignificant compared to the inhalation doses. The doses from resuspension are also shown to be negligible for the tank farm analysis conditions.

TRADEMARK DISCLAIMER. Reference herein to any specific commercial product, process, or service by trade name, trademark, manufacturer, or otherwise, does not necessarily constitute or imply its endorsement, recommendation, or favoring by the United states Goverment or any agency thereof or its contractors or subcontractors.

Printed in the United States of America. To obtain copies of this document, contact: WHC/BCS Document Control Services, P.0. Box 1970, Mailstop H6-08, Richland WA 99352, Phone (509) 372-2420; Fax (509) 376-4989.
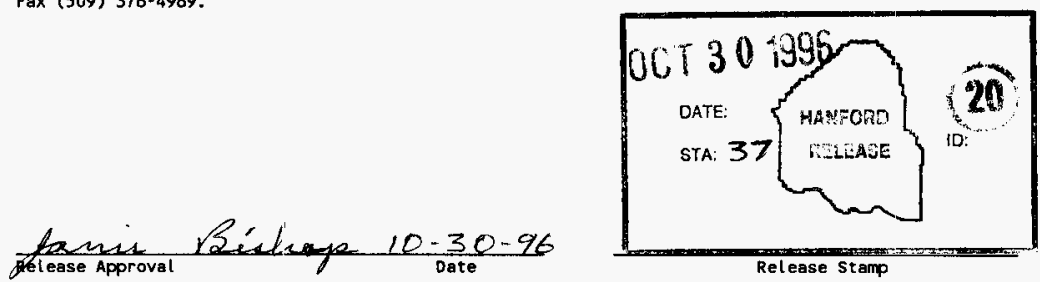

Approved for Public Release 


\section{INTRODUCTION}

The doses reported in the Tank Waste Remediation System (TWRS) Final Safety Analysis (FSAR) and the TWRS Basis of Interim Operation (BI0) are calculated from the inhalation and ingestion of the released material. Submersion doses, resuspension, and ground shine effects are not included. Detailed calculations which demonstrate that these doses are much less than the inhalation doses are given in this report. The relative contribution of submersion, resuspension, and ground shine are discussed in the following three sections.

\section{SUBMERSION}

The accident scenarios in the TWRS FSAR and BIO involve radioactive material from a Hanford waste tank being released to air and carried by the wind in a plume to a receptor. The principal dose to the receptor is calculated due to the radioactive materials being inhaled and retained in the body. However, in addition to the inhalation, the receptor will also receive an external dose from being submersed in the plume of radioactive material as it passes. The inhalation and submersion doses are compared in this section.

The submersion and inhalation doses for each tank farm composite was calculated at the onsite individual using the GENII code (PNL-1988). An onsite $X / Q$ of $3.41 \times 10^{-2}$ was taken from SARR-016 (WHC 1996a). Doses were calculated for each of six types of tank farm mixes, i.e., single shell tanks (SST) solids and liquids, double shell tank (DST) solids and liquids and aging waste facility (AWF) solids and liquids. The isotope mix for each composite was taken from WHC-SD-WM-SARR-016 (WHC 1996a) and are identical with those used in the TWRS FSAR and BIO. Submersion doses were calculated using an infinite cloud model. The GENII outputs are given in Attachment 1. The inhalation and submersion dose and the ratios are shown in Table 1.

Table 1 Comparison of Inhalation and Submersion Doses for Tank Farm Mixes at $100 \mathrm{~m}$ Onsite Receptor

\begin{tabular}{|l|c|c|c|}
\hline $\begin{array}{l}\text { Mixture of } \\
\text { Isotopes }\end{array}$ & \begin{tabular}{c} 
Inha $\begin{array}{c}\text { ation Dose } \\
(\mathrm{SV})\end{array}$ \\
\hline SST Solids
\end{tabular} & $\begin{array}{c}\text { Submersion Dose } \\
\text { (Sv) }\end{array}$ & $\begin{array}{l}\text { Submersion Dose } \\
\text { Divided by } \\
\text { Inhalation Dose }\end{array}$ \\
\hline SST Liquids & $1.2 \mathrm{E}+00$ & $1.5 \mathrm{E}-04$ & $6.5 \mathrm{E}-05$ \\
\hline DST Solids & $5.9 \mathrm{E}+00$ & $2.9 \mathrm{E}-05$ & $2.4 \mathrm{E}-04$ \\
\hline DST Liquids & $6.7 \mathrm{E}-02$ & $6.5 \mathrm{E}-05$ & $1.1 \mathrm{E}-05$ \\
\hline AWF Solids & $1.9 \mathrm{E}+01$ & $6.3 \mathrm{E}-05$ & $9.4 \mathrm{E}-04$ \\
\hline AWF Liquids & $1.5 \mathrm{E}-02$ & $1.8 \mathrm{E}-04$ & $9.5 \mathrm{E}-06$ \\
\hline
\end{tabular}


WHC-SD-WM-CN-080, Rev 0

The submersion dose is always a small fraction of the inhalation dose. Reactor accidents, which can result in a release of large quantities of radioactive krypton and xenon gases, can produce significant submersion doses. Inert gases give negligible inhalation doses but some of the inert gas isotopes have energetic gamma rays, which can produce significant submersion doses. These isotopes have short half-lives, and are not now present in the tank waste since the waste is a minimum of several year old. All the inert gases except for ${ }^{85} \mathrm{Kr}$ have decayed. ${ }^{85} \mathrm{Kr}$ emits only a weak beta, and does not contribute to submersion doses. The submersion doses for the tank farm mixes are dominated by the ${ }^{137} \mathrm{Cs}$ but the transuranic isotopes and ${ }^{90} \mathrm{Sr}$ produce inhalation doses which are much larger than the submersion doses.

Not including the submersion dose therefore does not affect the conclusions of the safety analysis.

\section{RESUSPENSION.}

The resuspension dose is the dose from material which falls out of the plume and is subsequently resuspended into the air due to wind or other activity. If resuspension is modeled, some faction of the material is assumed to fall to the ground, and the fraction of that material which is resuspended is calculated. Accident scenarios used for the TWRS FSAR do not assume that material from the plume is deposited on the ground prior to reaching the receptor. Concentrations in the plume decrease as the plume moves from the release point due to diffusion, but $100 \%$ of the material released is assumed to remain in the air and to reach both the onsite and offsite receptors.

Since any deposition/resuspension model would calculate less than $100 \%$ of the material reaches the receptor, the model used for the FSAR is conservative. A dose from resuspension can be calculated if deposition is assumed (although the fraction of material resuspended is usually small), but calculating an additional dose from resuspension does not make sense if no reduction in the dose for deposition is assumed.

An accident scenario that results in a pool formation and subsequent dry out can lead to doses from suspension of particles in the air. The releases from spills that result in pools do consider suspension of particles. This is a different accident scenario than a resuspension of deposited particles from a plume.

The spray leak events calculated doses are based on a respirable fraction of materials that are released by the spray. The larger particles could deposit on the ground near the spray source, and result in a pool. Particles could be released following the pool dry-out, as modeled for the spiti events. Significant releases would occur only for the unmitigated spray leak event. The analysis of the unmitigated spray leak event indicates that mitigation is required. Further refinement of the spray models to look at suspension from a pool would not change that conclusion. 
WHC-SD-WM-CN-080, Rev 0

Not including the plume deposition and resuspension dose therefore does not affect the conclusions of the safety analysis.

\section{GROUND SHINE}

Ground shine dose is the dose due to external exposure from material that deposit on the ground. The ground shine is compared to a inhalation for a specific scenario and the results extrapolated to a more general case.

A release of $0.001 \mathrm{~L} / \mathrm{s}$ of SST solids for 1 hour is assumed. SST solids is assumed because this composite has the largest concentrations of ${ }^{137} \mathrm{Cs}$ which tends to dominate shine calculations. The dose is calculated at the $100 \mathrm{~m}$ receptor.

The surface concentration of material on the ground from a passing plume is given by (PNL 1988):

$C_{i}=X v_{d} t$

where $C_{i}=$ surfaçe area concentration on radioactive isotope $i$ on the ground $\left(\mathrm{Bq} / \mathrm{m}^{2}\right)$

$X=$ concentration of material in the air $\left(\mathrm{Bq} / \mathrm{m}^{3}\right)$

$v_{d}=$ deposition velocity $(\mathrm{m} / \mathrm{s})$

$t=$ time of passage of plume over ground ( $s$ )

$X$ is the $X / Q^{\prime}$ times the release rate which is $0.001 \mathrm{~L} / \mathrm{s} \times 0.0341 \mathrm{~s} / \mathrm{m}^{3} \times G_{i}$ where $G_{i}$ is the activity concentration for the ith isotope in the mix in $B q / L$. The deposition velocity is a measure of the rate of material being deposited on the ground from the plume. The deposition velocity will depend on the particle size distribution but a velocity of $0.01 \mathrm{~m} / \mathrm{s}$ is generally considered bounding for respirable particles. The time, $t$ is assumed to be the time of release (1 hour).

The surface concentration on the ground for each isotope is therefore:

$$
\begin{aligned}
C_{i}\left(B q / \mathrm{m}^{2}\right) & =0.001 \mathrm{~L} / \mathrm{s} \times 0.0341 \mathrm{~s} / \mathrm{m}^{3} \times \mathrm{G}_{i} B q / \mathrm{L} \times 0.01 \mathrm{~m} / \mathrm{s} \times 3600 \mathrm{~s} \\
& =1.23 \times 10^{-3} \mathrm{G}_{\mathrm{i}}
\end{aligned}
$$

The $G_{i} s$ are taken from SARR-016 (WHC, 1996a). The $G_{i} s$ and the ground concentrations are showr in Table 2 . 
WHC-SD-WM-CN-080, Rev 0

Table 2 Surface Contamination Levels at $100 \mathrm{~m}$ for a $0.01 \mathrm{~s} / \mathrm{L}$ Release of SST solids

\begin{tabular}{|c|c|c|}
\hline Isotope & $\begin{array}{l}\mathrm{G}_{i} \text {, Concentration in } \\
\text { Composite }(\mathrm{Bg} / \mathrm{L})\end{array}$ & $\begin{array}{l}C_{i}, \quad \text { Surface } \\
\text { Contamination } \\
\text { Level }\left(\mathrm{Bg} / \mathrm{m}^{2}\right)\end{array}$ \\
\hline $\mathrm{Co}-60$ & $4.2 E+08$ & $5.17 \mathrm{E}+05$ \\
\hline $\mathrm{Cs}-137^{*}$ & $1.0 \mathrm{E}+11$ & $1.23 E+08$ \\
\hline Eu-154 & $5.8 E+09$ & $7.13 \mathrm{E}+06$ \\
\hline $\mathrm{Eu}-155$ & $5.0 E+06$ & $6.15 E+03$ \\
\hline
\end{tabular}

* The Cs-137 daughter Ba-137m is assumed to be present at 0.946 of the Cs-137 concentration. $(0.946$ is the branching ratio for the decay of $\mathrm{Cs}-137$ to $\mathrm{Ba}-$ $137 \mathrm{~m})$.

The ground shine is dependent on the area of ground contamination which in turn depends on how wide the plume has spread by the time it has reached the vicinity of the receptor. A plume width based on gaussian diffusion can be calculated based on a quantity referred to as $\sigma_{y}$ for the $p l$ ume model. The $X / Q$ is based on F stability. The formula for $\sigma_{y}$ for $F$ stability is taken from PNL 1988 and is given by:

$\sigma_{y}=A_{y} x^{B y}$

where $A_{y}=0.0722$ and $B_{y}=0.9031$.

The $\sigma_{y}=4.62 \mathrm{~m}$ at $100 \mathrm{~m}$. The gaussian plume theoretical width in the transverse direction is infinite but the majority of the material will be contained in a finite width. If the amount of material were to be packed into an interval such that the concentration is uniform and equal to the maximum concentration the width is $(2 \pi)^{1 / 2} \sigma_{y}$. The plume width is $(2 \pi)^{1 / 2} 4.62=11.58$ $\mathrm{m}$. The contamination will be assumed to be spread over an area with this width. The plume length will be assumed to extend $20 \mathrm{~m}$ towards the source and $20 \mathrm{~m}$ away from the source $(40 \mathrm{~m}$ total). The contamination extends to infinity but a calculation assuming $10 \mathrm{~m}$ in length in each direction (instead of $20 \mathrm{~m}$ ) produced only a $6 \%$ difference in dose rate, so the use of $20 \mathrm{~m}$ is a good approximation of the length of the contamination that contributes to the gamma ray doses. The concentration and surface contamination are calculated using equation 1.

The doses are computed based on a rectangular source area around the receptor with dimensions of $40 \mathrm{~m}$ by $11.58 \mathrm{~m}$. The dose is calculated at the midpoint of rectangle at a height of $1 \mathrm{~m}$ above the ground. The surface concentrations in Table 2 are multiplied by $463 \mathrm{~m}^{2}$ to produce a total source term. The surface concentration was modeled as a uniform layer $0.001 \mathrm{~m}$ thick. An additional correction is applied to compensate for the fact that the concentration will be higher toward the source than away from it. $X / Q \mathrm{~s}$ at $80 \mathrm{~m}$ and $120 \mathrm{~m}$ in the east direction were calculated using the GXQ code (Hey 1993). The east 
direction produces the maximum $X / Q$ (WHC 1996a). The GXQ output is shown in attachment 2. The $X / Q$ at $80 \mathrm{~m}$ is $0.0499 \mathrm{~s} / \mathrm{m}^{3}$ and at $120 \mathrm{~m}$ is $0.0248 \mathrm{~s} / \mathrm{m}^{3}$. The average $X / Q$ is therefore is $0.0374 \mathrm{~s} / \mathrm{m}^{3}$. The total activities were increased by $0.0374 / 0.0341=1.1$ to compensate for this non-linearity in the atmospheric dispersion coefficients.

The total activity deposited on the ground is given in Table 3 for each isotope.

Table 3 Total Activities for Ground Shine Calculation.

\begin{tabular}{||l|c|}
\hline Isotope & $\begin{array}{c}\text { Total activities } \\
\mathrm{Bq}\end{array}$ \\
\hline $\mathrm{Co}-60$ & $2.63 \mathrm{E}+08$ \\
\hline $\mathrm{Cs}-137 *$ & $6.27 \mathrm{E}+10$ \\
\hline $\mathrm{Eu}-154$ & $3.63 \mathrm{E}+09$ \\
\hline Eu-155 & $3.13 \mathrm{E}+06$ \\
\hline
\end{tabular}

* The Cs-137 daughter $\overline{\mathrm{Ba}}-\sqrt{37 \mathrm{~m}}$ is assumed to be present at 0.946 the $\mathrm{Cs}-137$ concentration. ( 0.946 is the branching ratio for the decay of Cs-137 to Ba$137 \mathrm{~m})$.

The dose rate from the ground contamination was calculated with MICROSHIELD Version 3 (Grove 1988). The computer code output file is given in Attachment 3. The dose rate is $20.4 \mathrm{mr} / \mathrm{hr}\left(2.04 \times 10^{-04} \mathrm{~Sv} / \mathrm{hr}\right)$. The receptor is assumed to remain at the worst case location for 12 hours. The ground contamination would continue to produce a dose rate after the 12 hours but people are assumed to be removed from the contaminated area involved after 12 hours. For a 12 hour exposure, the dose is $2.45 \times 10^{-3} \mathrm{~Sv}$.

The inhalation dose for this accident scenario is given by (WHC 1996a):

$D=Q \times X / Q^{\prime} \times B R \times U L D$

where $D=$ Inhalation dose (Sv)

$Q=$ Release quantity $=0.001 \mathrm{~L} / \mathrm{s} \times 3600 \mathrm{~s}=3.6 \mathrm{~L}$

$X / Q^{\prime}=0.0341 \mathrm{~s} / \mathrm{m}^{3}$

$B R=$ Breathing rate $=3.3 \times 10^{-4} \mathrm{~m}^{3} / \mathrm{s}$ for light activity

ULD $=2.2 \times 10^{5} \mathrm{~Sv} / \mathrm{L}$ (from SARR-016, WHC, 1996a)

The inhalation dose is $8.91 \mathrm{~Sv}$.

The ground shine dose divided by the inhalation dose is therefore:

$2.45 \times 10^{-3} \mathrm{~Sv} / 8.91 \mathrm{SV}=2.75 \times 10^{-4}$.

This calculation was made for an arbitrary release of $0.001 \mathrm{~s} / \mathrm{L}$. However it can be seen from equations 1 and 3 that both the inhalation and ground shine 
WHC-SD-WM-CN-080, Rev 0

doses are directly proportional to the release rate, and thus the conclusion that the ground shine dose is insignificant relative to inhalation dose would apply for all release rates. Both doses are directly proportion to the $X / Q$, so the conclusion would also apply at all receptor locations. The composite picked (SST solids) is the worst case composite since it is relatively high in gamma emitter, and relatively low in transuranics which contribute heavily to the inhalation dose calculation, so this conclusion would also apply to the other composites. It is possible to look at more detailed modelling of the ground contamination (for instances looking at different stability classes), but the correction will not affect the conclusion that the ground

contamination dose is much less than the inhalation dose, since assumptions are conservative, and the results indicate that the ground shine dose is over three orders of magnitude less than the inhalation doses.

The dose from inhalation assumes that the particles inhaled are of a respirable size. The dose from ground shine can be produced by particles of larger than respirable size. However, particles of larger than respirable size tend to drop out of the plume very quickly, and are not transported to the vicinity of the receptor. Thus particles of larger than respirable size will not contribute significantly to the ground shine dose for the onsite or offsite individual. Elevated releases can result in longer range transport of larger particles, but the tank farm doses are based on ground level releases.

Not including the ground shine doses therefore does not affect the conclusions of the safety analysis.

\section{REFERENCES}

Grove 1988, MICROSHIELD Version 3, Grove Engineering Inc, 15125 Shady Grove Road, Rockville, Maryland.

Hey, B. E., 1993, GXQ Program Users Guide, WHC-SD-GN-SWD-3002, Westinghouse Hanford Company, Richland, Wa.

PNL, 1988, B. A. Napier, et al, GENII - The Hanford Environmental Radiation Dosimetry Software System, Volume 1: Conceptual Representation, PNL-6584, Pacific Northwest Laboratory, Richland Wa.

WHC, 1996a, J.C. Van Keuren, Tank Waste Compositions and Atmospheric Dispersion Coefficients for Use in Safety Analysis Consequence Assessments, WHC-SD-WM-SARR-016, Rev 2, Richland Wa. 
WHC-SD-WM-CN-080, Rev 0

Attachment 1 GENII output files 


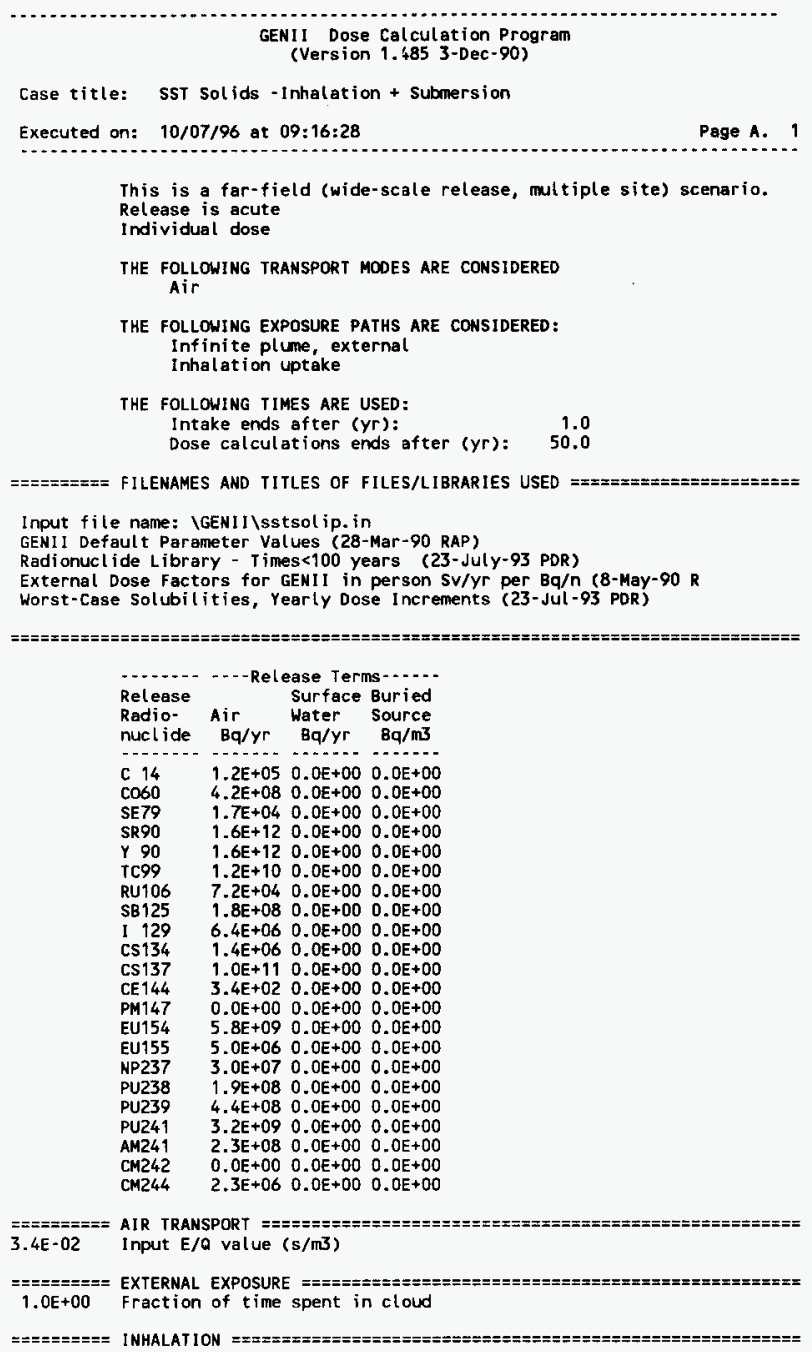


WHC-SD-WM-CN-080, Rev 0

Resuspension not considered

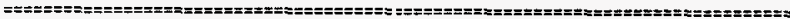

Input prepared by:

Date:

Input checked by:

Date: 


\section{WHC-SD-WM-CN-080, Rev 0}

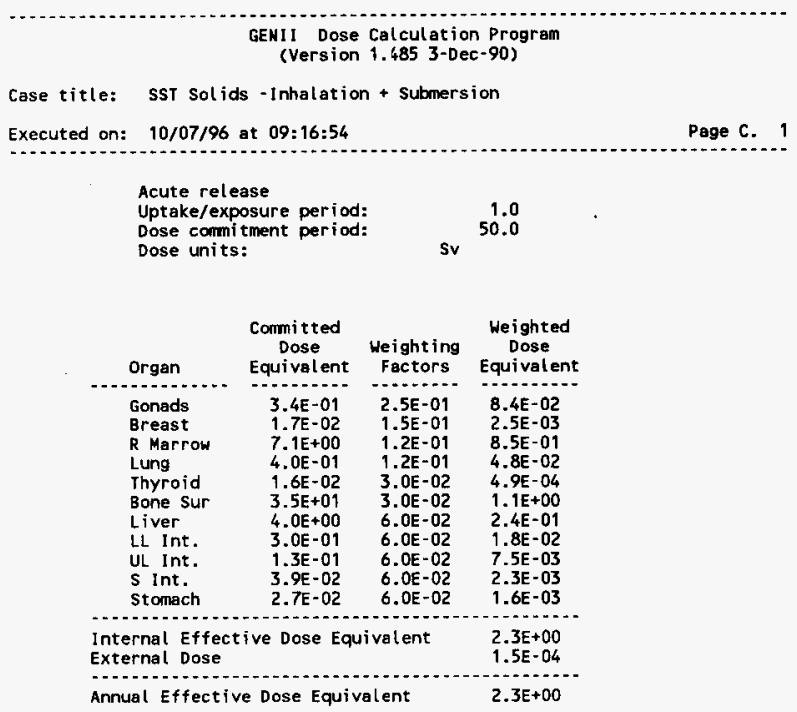

\begin{tabular}{|c|c|}
\hline $\begin{array}{l}\text { Controlling Organ: } \\
\text { Controll ing Pathway: } \\
\text { Controll ing Radionucl ide: }\end{array}$ & $\begin{array}{l}\text { Bone Sur } \\
\text { Inh } \\
\text { SR } 90\end{array}$ \\
\hline $\begin{array}{l}\text { Total Inhalation EDE: } \\
\text { Total Ingest ion EDE: }\end{array}$ & $\begin{array}{l}2.3 \mathrm{E}+00 \\
0.0 \mathrm{E}+00\end{array}$ \\
\hline
\end{tabular}


WHC-SD-WM-CN-080, Rev 0

GENII Dose Calculation Program

(version 1. 485 3-Dec-90)

Case title: SST Solids -Inhalation + Submersion

Executed on: 10/07/96 at 09:16:54

Page C. 2

Acute release

Uptake/exposure period:

Dose commitment period:

Dose units:

Sv $\begin{array}{r}1.0 \\ 50.0\end{array}$

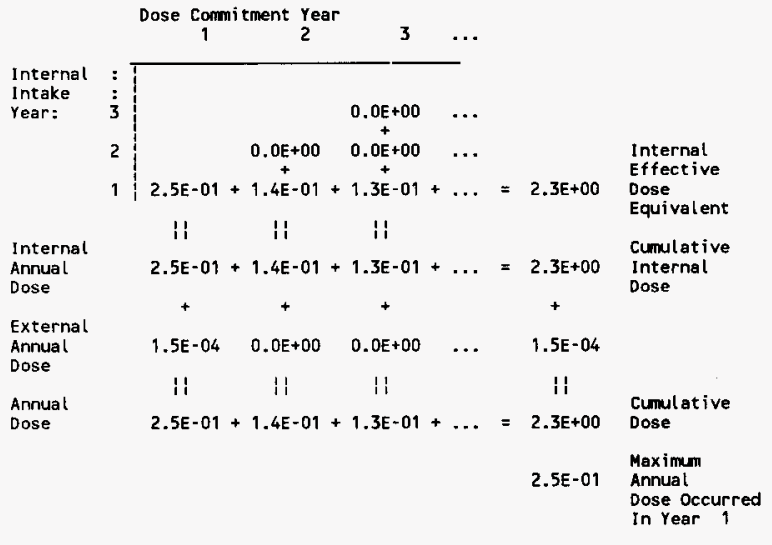




\section{WHC-SD-WM-CN-080, Rev 0}

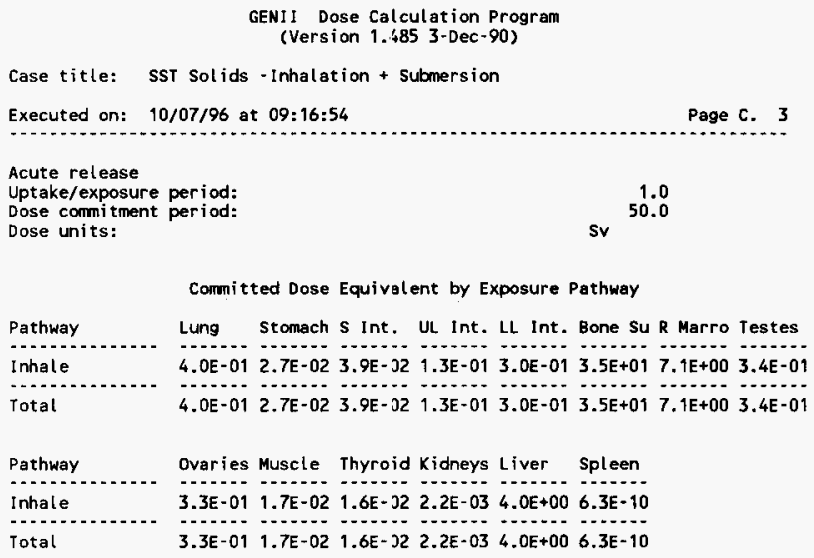

External Dose by Exposure Pathway

Pathway

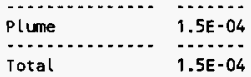


WHC-SD-WM-CN-080, Rev 0

GENII Dose Calculation Program

(Version 1.485 3-Dec-90)

Case title: SST Solids -Inhalation + Submersion

Executed on: 10/07/96 at 09:16:54

Page C. 4

Acute release

Uptake/exposure period:

Dose commitment period:

Dose units:

sv $\begin{array}{r}1.0 \\ 50.0\end{array}$

Committed Dose Equivalent by Radionuclide

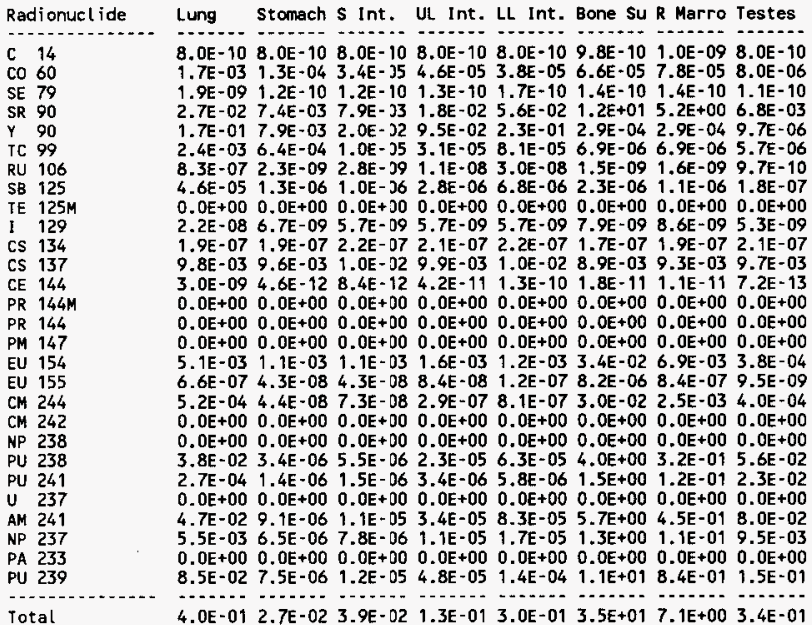

GENII Dose Calculation Program

(Version 1.485 3-Dee-90)

Case title: SST Solids - Inhalation + Submersion

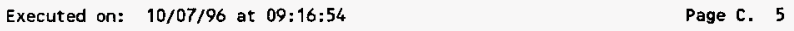

Acute release

Uptake/exposure period:

Dose commitment period:

Dose units:

Sv $\begin{array}{r}1.0 \\ 50.0\end{array}$

1.0

Committed Dose Equivalent by Radionucl ide

Radionuclide

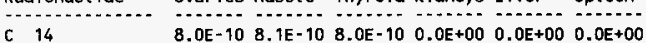

c 14

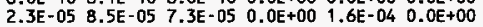




\section{WHC-SD-WM-CN-080, Rev 0}

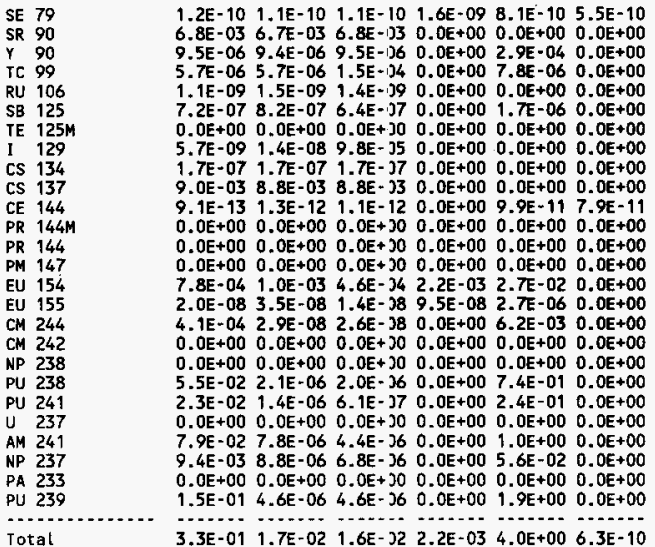




\section{WHC-SD-WM-CN-080, Rev 0}

\begin{tabular}{|c|c|c|c|c|c|}
\hline \multirow[t]{2}{*}{$\begin{array}{l}\text { ase title: } \\
\text { xecuted on: } \\
\end{array}$} & \multicolumn{5}{|c|}{ SST Solids - Inhalation + submersion } \\
\hline & $\begin{array}{l}\text { Acute relea } \\
\text { Uptake/expo } \\
\text { Dose commit } \\
\text { Dose units: }\end{array}$ & $\begin{array}{l}\text { se } \\
\text { sure period: } \\
\text { ment period: }\end{array}$ & Sv & $\begin{array}{l}1.0 \\
0.0\end{array}$ & \\
\hline $\begin{array}{l}\text { Radio- } \\
\text { nuclide }\end{array}$ & $\begin{array}{c}\text { Inhalation } \\
\text { Ef fective } \\
\text { Dose } \\
\text { Equivalent }\end{array}$ & $\begin{array}{l}\text { Ingestion } \\
\text { Effective } \\
\text { Dose } \\
\text { Equivalent }\end{array}$ & $\begin{array}{l}\text { External } \\
\text { Dose }\end{array}$ & $\begin{array}{l}\text { Internel } \\
\text { Effective } \\
\text { Dose } \\
\text { Equivalent }\end{array}$ & $\begin{array}{l}\text { Annusl } \\
\text { Effective } \\
\text { Dose } \\
\text { Equivalent }\end{array}$ \\
\hline $\begin{array}{ll}\text { C } & 14 \\
\text { CO } & 60 \\
\text { SE } & 79 \\
\text { SR } & 90 \\
\text { Y } & 90 \\
\text { IC } & 99 \\
\text { RU } & 106 \\
\text { SB } & 125 \\
\text { IE } & 125 M \\
\text { I } & 129 \\
\text { CS } & 134 \\
\text { CS } & 137 \\
\text { CE } & 144 \\
\text { PR } & 144 M \\
\text { PR } & 144 \\
\text { PM } & 147 \\
\text { EU } & 154 \\
\text { EU } & 155 \\
\text { CM } & 244 \\
\text { CM } & 242 \\
\text { NP } & 238 \\
\text { PU } & 238 \\
\text { PU } & 241 \\
\text { U } & 237 \\
\text { AM } & 241 \\
\text { NP } & 237 \\
\text { PA } & 233 \\
\text { PU } & 239\end{array}$ & $\begin{array}{l}7.8 E-10 \\
2.6 E-04 \\
4.9 E-10 \\
9.9 E-01 \\
4.2 E-02 \\
3.4 E-04 \\
1.0 E-07 \\
6.9 E-06 \\
0.0 E+00 \\
2.9 E-06 \\
1.8 E-07 \\
8.9 E-03 \\
3.8 E-10 \\
0.0 E+00 \\
0.0 E+00 \\
0.0 E+00 \\
4.8 E-03 \\
6.2 E-07 \\
1.7 E-03 \\
0.0 E+00 \\
0.0 E+00 \\
2.2 E-01 \\
8.0 E-02 \\
0.0 E+00 \\
3.1 E-01 \\
5.8 E-02 \\
0.0 E+00 \\
5.9 E-01\end{array}$ & $\begin{array}{l}0.0 \mathrm{E}+00 \\
0.0 \mathrm{E}+00 \\
0.0 \mathrm{E}+00 \\
0.0 \mathrm{E}+00 \\
0.0 \mathrm{E}+00 \\
0.0 \mathrm{E}+00 \\
0.0 \mathrm{E}+00 \\
0.0 \mathrm{E}+00 \\
0.0 \mathrm{E}+00 \\
0.0 \mathrm{E}+00 \\
0.0 \mathrm{E}+00 \\
0.0 \mathrm{E}+00 \\
0.0 \mathrm{E}+00 \\
0.0 \mathrm{E}+00 \\
0.0 \mathrm{E}+00 \\
0.0 \mathrm{E}+00 \\
0.0 \mathrm{E}+00 \\
0.0 \mathrm{E}+00 \\
0.0 \mathrm{E}+00 \\
0.0 \mathrm{E}+00 \\
0.0 \mathrm{E}+00 \\
0.0 \mathrm{E}+00 \\
0.0 \mathrm{E}+00 \\
0.0 \mathrm{E}+00 \\
0.0 \mathrm{E}+00 \\
0.0 \mathrm{E}+00 \\
0.0 \mathrm{E}+00 \\
0.0 \mathrm{E}+00\end{array}$ & $\begin{array}{l}1.8 \mathrm{E}-15 \\
1.9 \mathrm{E}-06 \\
1.9 \mathrm{E}-16 \\
6.0 \mathrm{E}-07 \\
2.2 \mathrm{E}-05 \\
8.6 \mathrm{E}-10 \\
3.2 \mathrm{E}-11 \\
1.6 \mathrm{E}-07 \\
0.0 \mathrm{E}+00 \\
1.1 \mathrm{E}-10 \\
4.4 \mathrm{E}-09 \\
1.1 \mathrm{E}-04 \\
9.0 \mathrm{E}-15 \\
0.0 \mathrm{E}+00 \\
0.0 \mathrm{E}+00 \\
0.0 \mathrm{E}+00 \\
1.3 \mathrm{E}-05 \\
3.9 \mathrm{E}-10 \\
5.9 \mathrm{E}-13 \\
0.0 \mathrm{E}+00 \\
0.0 \mathrm{E}+00 \\
5.7 \mathrm{E}-11 \\
2.3 \mathrm{E}-15 \\
0.0 \mathrm{E}+00 \\
4.7 \mathrm{E}-09 \\
7.8 \mathrm{E}-10 \\
0.0 \mathrm{E}+00 \\
8.7 \mathrm{E}-11\end{array}$ & $\begin{array}{l}7.8 \mathrm{E}-10 \\
2.6 \mathrm{E}-04 \\
4.9 \mathrm{E}-10 \\
9.9 \mathrm{E}-01 \\
4.2 \mathrm{E}-02 \\
3.4 \mathrm{E}-04 \\
1.0 \mathrm{E}-07 \\
6.9 \mathrm{E}-06 \\
0.0 \mathrm{E}+00 \\
2.9 \mathrm{E}-06 \\
1.8 \mathrm{E}-07 \\
8.9 \mathrm{E}-03 \\
3.8 \mathrm{E}-10 \\
0.0 \mathrm{E}+00 \\
0.0 \mathrm{E}+00 \\
0.0 \mathrm{E}+00 \\
4.8 \mathrm{E}-03 \\
6.2 \mathrm{E}-07 \\
1.7 \mathrm{E}-03 \\
0.0 \mathrm{E}+00 \\
0.0 \mathrm{E}+00 \\
2.2 \mathrm{E}-01 \\
8.0 \mathrm{E}-02 \\
0.0 \mathrm{E}+00 \\
3.1 \mathrm{E}-01 \\
5.8 \mathrm{E}-02 \\
0.0 \mathrm{E}+00 \\
5.9 \mathrm{E}-01\end{array}$ & $\begin{array}{l}7.8 \mathrm{E}-10 \\
2.6 \mathrm{E}-04 \\
4.9 \mathrm{E}-10 \\
9.9 \mathrm{E}-01 \\
4.2 \mathrm{E}-02 \\
3.4 \mathrm{E}-04 \\
1.0 \mathrm{E}-07 \\
7.0 \mathrm{E}-06 \\
0.0 \mathrm{E}+00 \\
2.9 \mathrm{E}-06 \\
1.9 \mathrm{E}-07 \\
9.0 \mathrm{E}-03 \\
3.8 \mathrm{E}-10 \\
0.0 \mathrm{E}+00 \\
0.0 \mathrm{E}+00 \\
0.0 \mathrm{E}+00 \\
4.9 \mathrm{E}-03 \\
6.2 \mathrm{E}-07 \\
1.7 \mathrm{E}-03 \\
0.0 \mathrm{E}+00 \\
0.0 \mathrm{E}+00 \\
2.2 \mathrm{E}-01 \\
8.0 \mathrm{E}-02 \\
0.0 \mathrm{E}+00 \\
3.1 \mathrm{E}-01 \\
5.8 \mathrm{E}-02 \\
0.0 \mathrm{E}+00 \\
5.9 \mathrm{E}-01\end{array}$ \\
\hline & & & & & \\
\hline
\end{tabular}




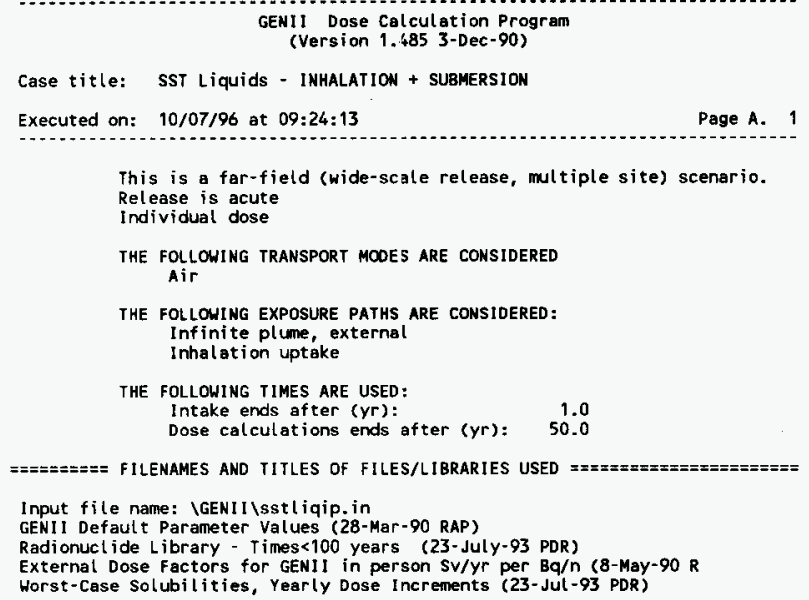

THE FOLLOWING TIMES ARE USED:

Intake ends af ter $(y r)$ : $\quad 1.0$ Dose calculations ends after $(y r): \quad 50.0$

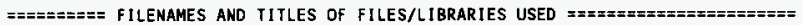

Input file name: IGENIIIsstliqip.in

GENII Defaut $t$ Parameter Values (28-Mar-90 RAP)

Radionuctide Library - Times< 100 years (23-July-93 PDR)

External Dose Factors for GENII in person Sv/yr per Bq/n (8-May-90 R

Worst-Case Solubilities, Yearly Dose Increments (23-Jut-93 PDR)

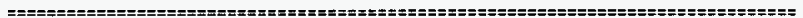

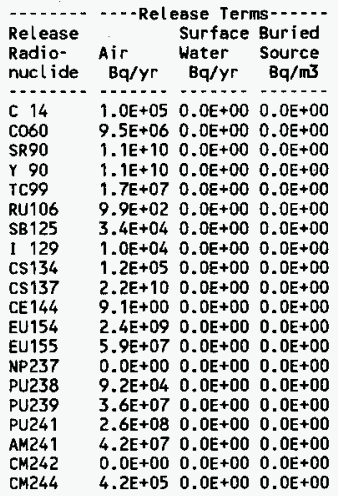

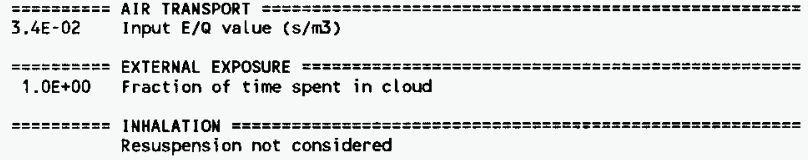


WHC-SD-WM-CN-080, Rev 0

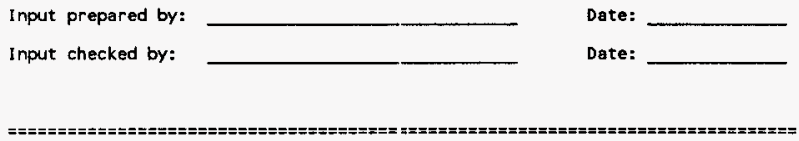


WHC-SD-WM-CN-080, Rev 0

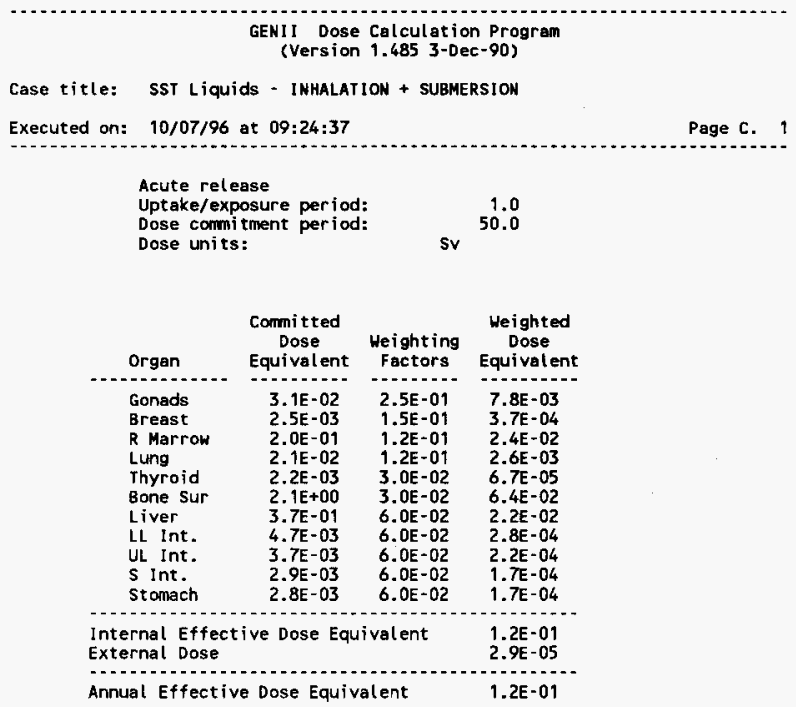

\begin{tabular}{|c|c|}
\hline $\begin{array}{l}\text { Controlling Organ: } \\
\text { Controlling Pathway: } \\
\text { Controll ing Radionucl ide: }\end{array}$ & $\begin{array}{l}\text { Bone Sur } \\
\text { Inh } \\
\text { AM241 }\end{array}$ \\
\hline $\begin{array}{l}\text { Total Inhalation EDE: } \\
\text { Total Ingestion EDE: }\end{array}$ & $\begin{array}{l}1.2 E- \\
0.0 E+\end{array}$ \\
\hline
\end{tabular}




\section{WHC-SD-WM-CN-080, Rev 0}

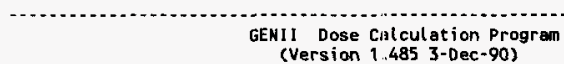

Case title: SST Liquids - INHALATIOII + SUBMERSION

Executed on: 10/07/96 at 09:24:37

Page C. 2

Acute release

Uptake/exposure period:

Dose commitment period:

Dose units:

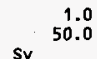

Sv
Internal

Equivalent

cumulative

il

Internal

Annual

Dose

External

Annual

Dose

Annual

Dose

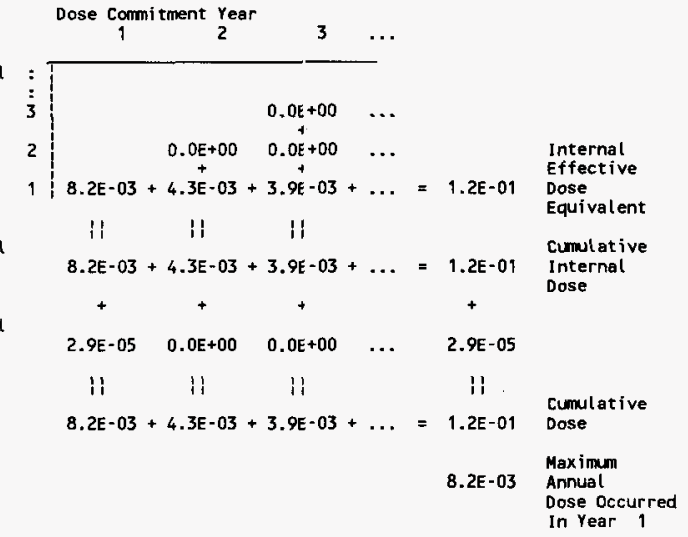




\section{WHC-SD-WM-CN-080, Rev 0}

GEN11 Dose Calculation Program
(Version 1.485 3-Dec-90)




\section{WHC-SD-WM-CN-080, Rev 0}

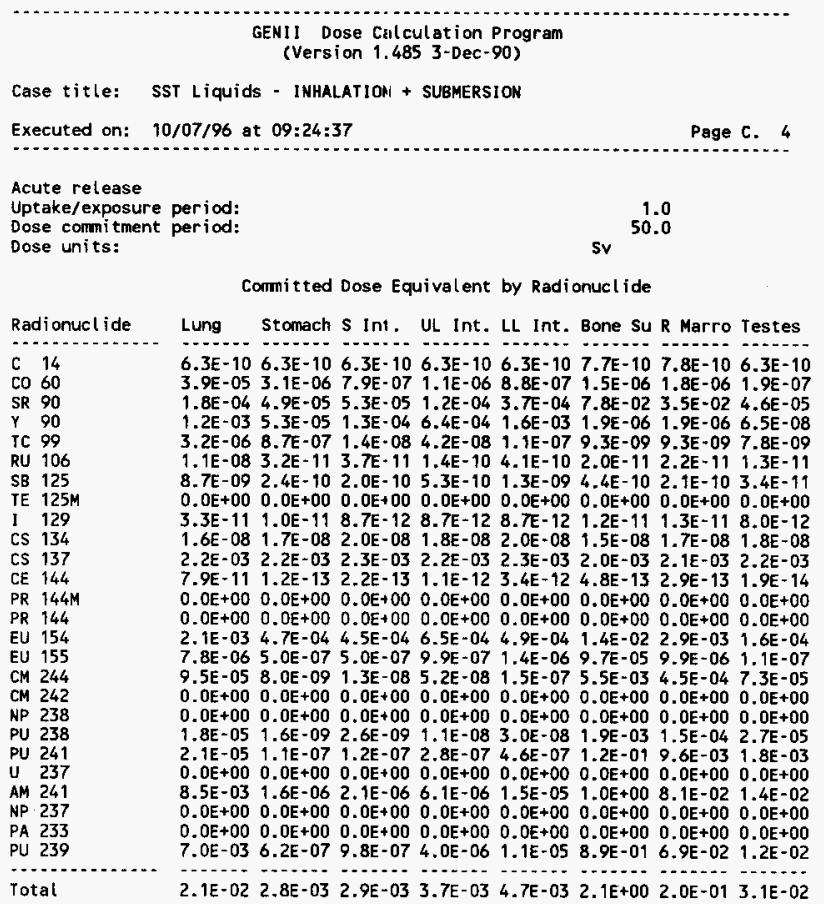




\section{WHC-SD-WM-CN-080, Rev 0}

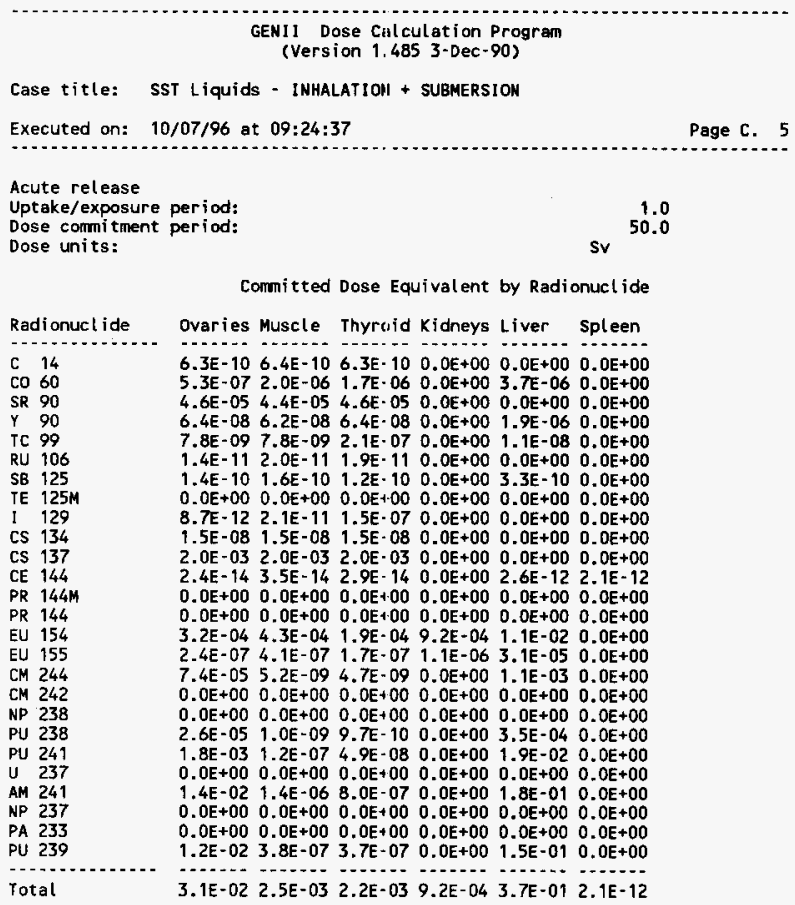




\section{WHC-SD-WM-CN-080, Rev 0}

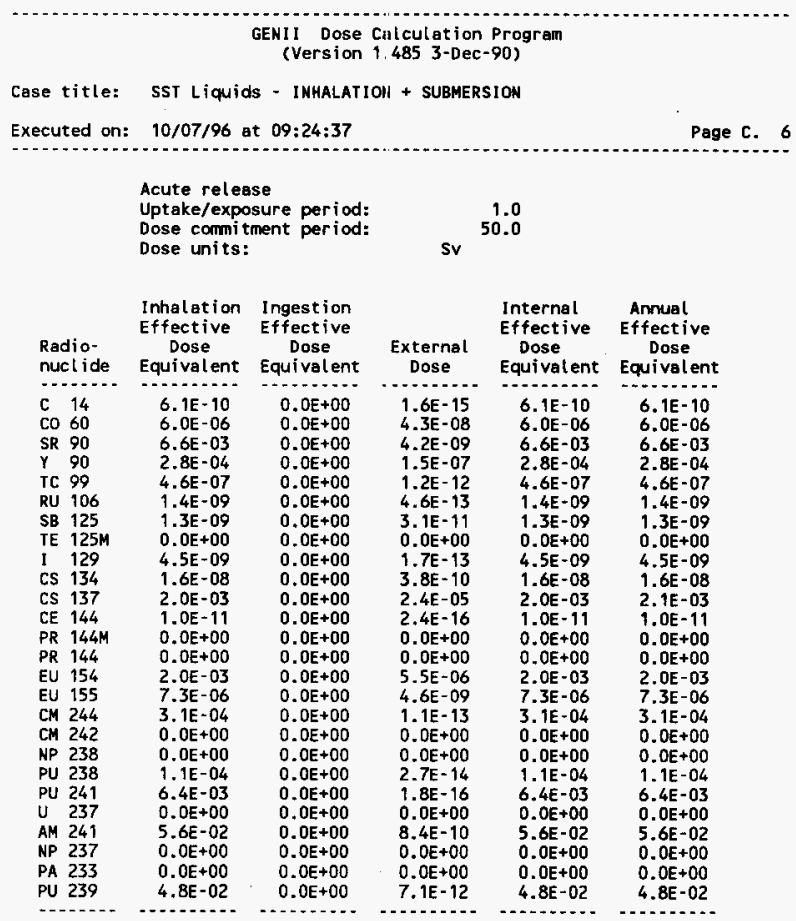


GENII Dose Calculation Program

(Version 1.485 3-Dec-90)

Case title: DST Solids - Inhalation + submersion.

Executed on: 10/07/96 at 09:33:07

Page A. 1

This is a far-field (wide-scale release, multiple site) scenario. Release is acute

Individual dose

THE FOLLONING TRANSPORT MOOES ARE CONSIDERED

Air

THE FOLLOWING EXPOSURE PATHS ARE CONSIDERED:

Infinite plume, external

Inhalation uptake

THE FOLLOWING TIMES ARE USE[':

Intake ends after (yr):

Dose calculations ends after $(y r): \quad 50.0$

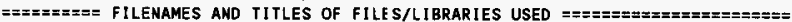

Input file name: IGENIIIdstsolip.in

GENII Default Parameter Values (28-Mar-90 RAP)

Radionuclide Library - Times $<100$ years (23-July-93 PDR)

External Dose Factors for GENIt in person SV/Yr per Bq/n (8-May-90 R

Worst-Case Solubilities, Yearly Dose Increments (23-Jul-93 PDR)

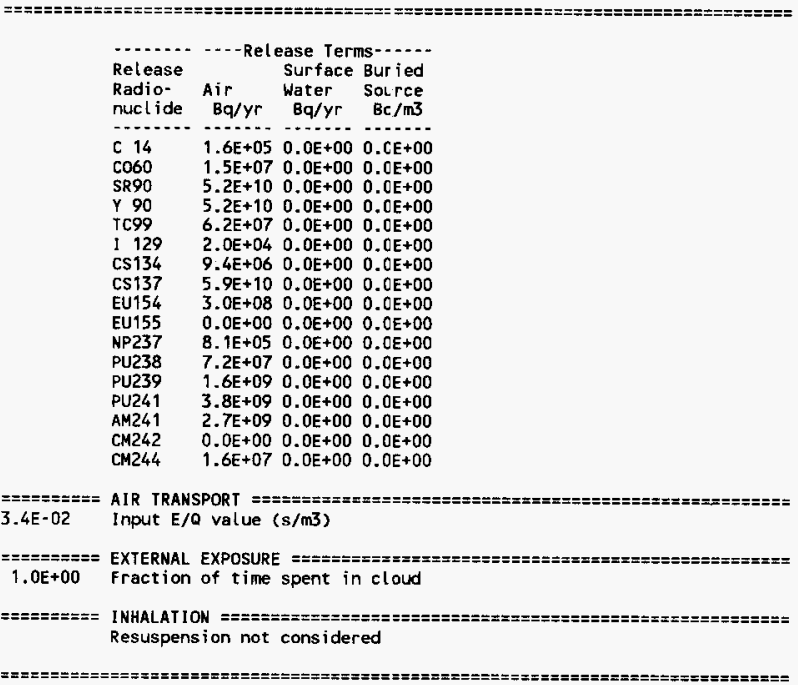


WHC-SD-WM-CN-080, Rev 0

Input prepared by:

Input checked by:
Date:

Date: 
WHC-SD-WM-CN-080, Rev 0

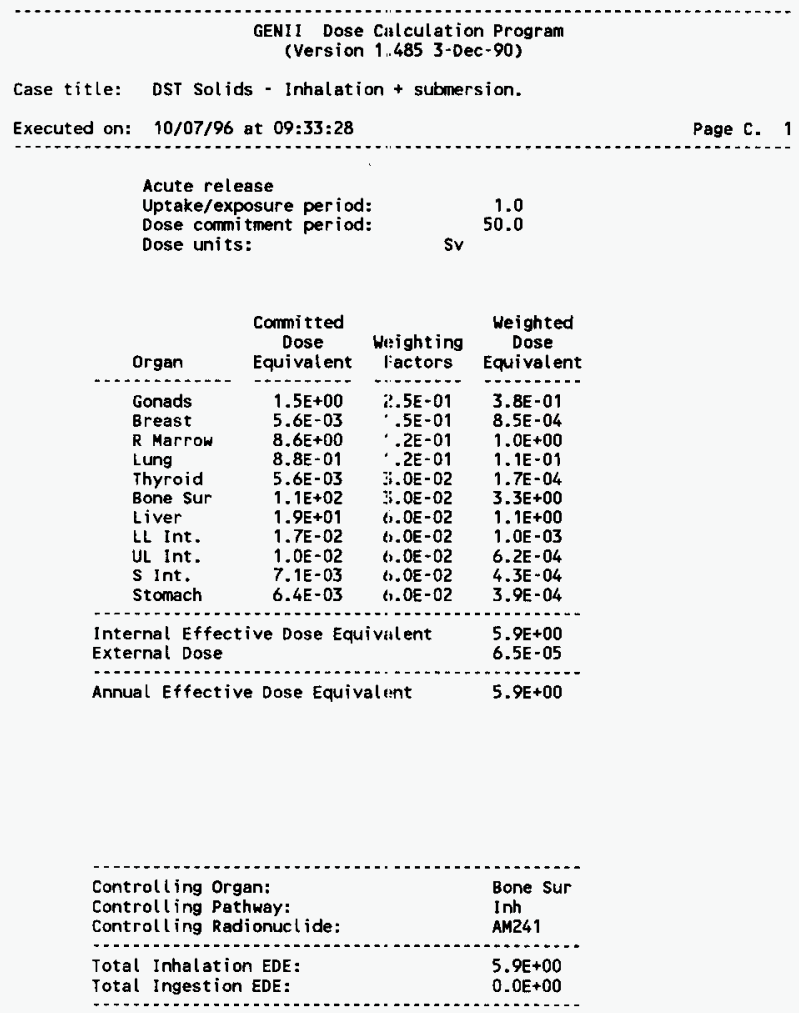


WHC-SD-WM-CN-080, Rev 0

GENII Dose Calculation Program

(Version 9 , 485 3-Dec-90)

Case title: DST Solids - Inhalation + submersion.

Executed on: 10/07/96 at 09:33:28

Page C. 2

Acute release

Uptake/exposure period:

Dose commitment period:

Dose units :

sv $\begin{array}{r}1.0 \\ 50.0\end{array}$

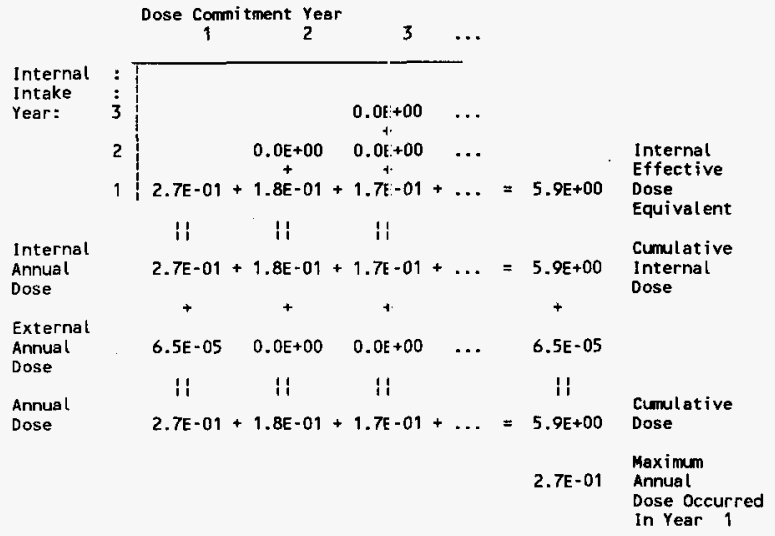




\section{WHC-SD-WM-CN-080, Rev 0}

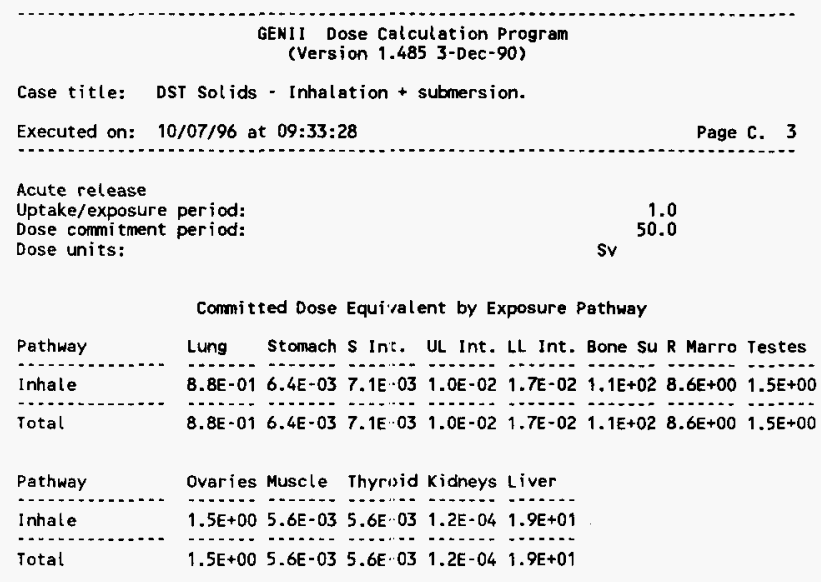

External Dose by Exposure Pathway

Pathway

\begin{tabular}{ll} 
& \\
\hline Plume & $6.5 \mathrm{E}-05$ \\
\hdashline Total & $6.5 \mathrm{E}-05$
\end{tabular}




\section{WHC-SD-WM-CN-080, Rev 0}

GENII Dose Calculation Program

(Version $1.4853-$ Dec-90)

Case title: DST Solids - Inhalation + submersion.

Executed on: 10/07/96 at 09:33:28

Page C. 4

Acute release

Uptake/exposure period:

Dose commitment period:

Dose units:

1.0
50.0

SV

Comitted Dose Equivalent by Radionucl ide

\begin{tabular}{ll} 
Radionuclide \\
\hline C 14 \\
CO & 60 \\
SR & 90 \\
$Y$ & 90 \\
TC & 99 \\
I & 129 \\
CS & 134 \\
CS & 137 \\
EU & 154 \\
EU 155 \\
CM 244 \\
CM 242 \\
NP 238 \\
PU 238 \\
PU 241 \\
U 237 \\
AM 241 \\
NP 237 \\
PA 233 \\
PU 239 \\
\hline
\end{tabular}

Total
Lung Stonach $\mathbf{S}$ Int. UL. Int. LL Int. Bone SU R Marro Testes

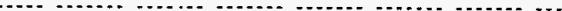
1.0E-09 1.0E-09 1.0E-09 1.0E-09 1.0E-09 1.3E-09 $1.3 \mathrm{E}-09$ 1.0E-09 6.0E-05 4.8E-06 1.2E-06 1.6E-06 1.4E-06 2.4E-06 2.8E-06 2.9E-07 8.8E-04 2.4E-04 2.6E-04 5.9E-04 1.8E-03 3.8E-01 1.7E-01 2.2E-04 5.7E-03 2.6E-04 6.5E-04 3.1E-03 7.7E-03 9.4E-06 9.4E-06 3.2E-07 1.2E-05 3.2E-06 5.0E-08 1.5E-07 4.1E-07 3.4E-08 3.4E-08 2.9E-08 6.9E-11 2.1E-11 $1.8 \mathrm{E}-11 \quad 1.8 \mathrm{E}-11 \quad 1.8 \mathrm{E}-11 \quad 2.5 \mathrm{E}-11 \quad 2.8 \mathrm{E}-11 \quad 1.7 \mathrm{E}-11$

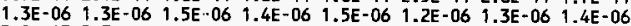
5.9E-03 5.7E-03 6.0E-03 5.9E-03 6.0E-03 5.3E-03 5.6E-03 $5.8 \mathrm{E}-03$

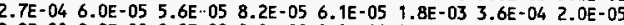

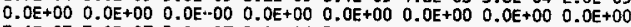
3.6E-03 3.1E-07 5.0E-07 2.0E-06 5.6E-06 2.1E-01 $1.7 \mathrm{E}-02 \quad 2.8 \mathrm{E}-03$

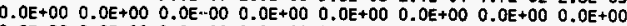

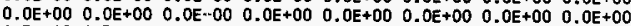
1.5E-02 1.3E-06 2.1E-06 B.9E-06 2.4E-05 1.6E+00 1.2E-01 2.1E-02

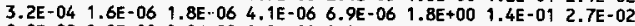

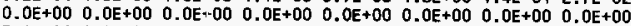

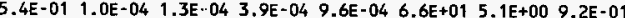
1.5E-04 1.7E-07 2.1E-07 2.9E-07 4.6E-07 3.5E-02 2.8E-03 2.6E-04

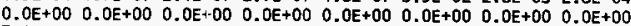
3. $1 \mathrm{E}-01$ 2.7E-05 4.3E-05 1.7E-04 5.0E-04 3.9E+01 3.0E+00 5.4E-01

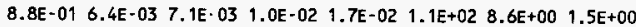




\section{WHC-SD-WM-CN-080, Rev 0}

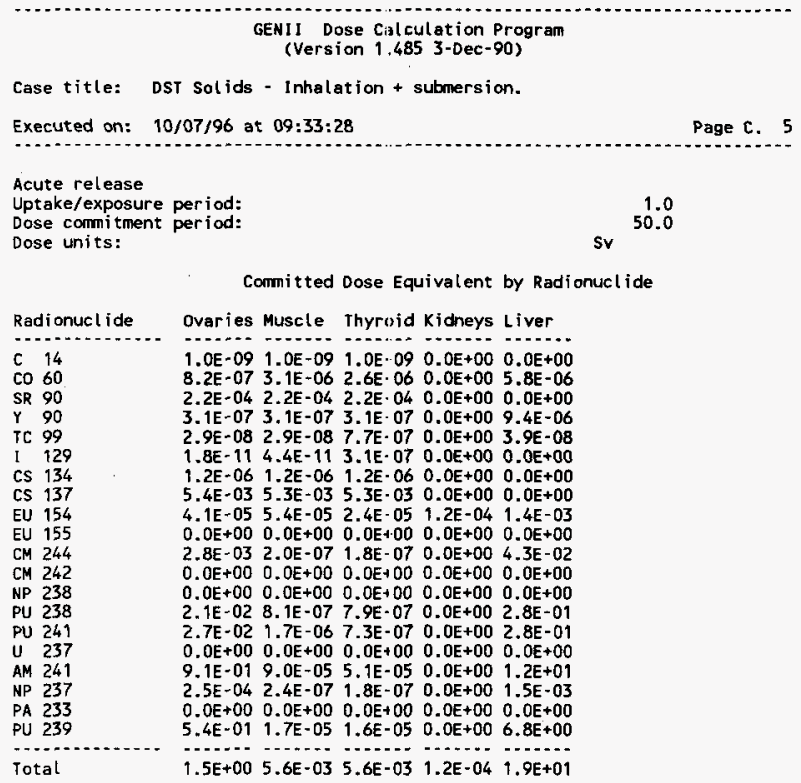




\section{WHC-SD-WM-CN-080, Rev 0}

GENII Dose Cialculation Program

(Version 1.485 3-Dec-90)

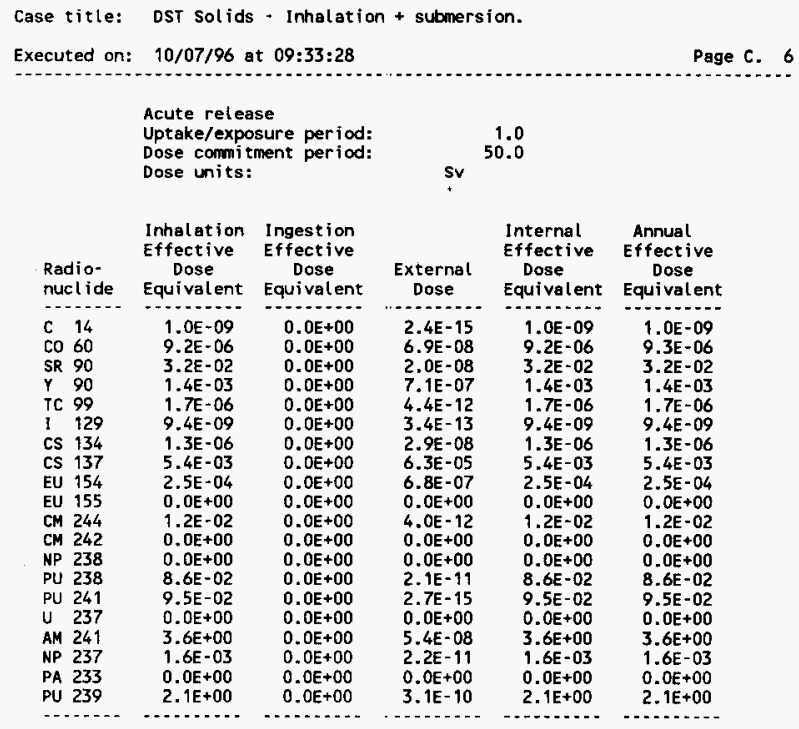




\section{WHC-SD-WM-CN-080, Rev 0}

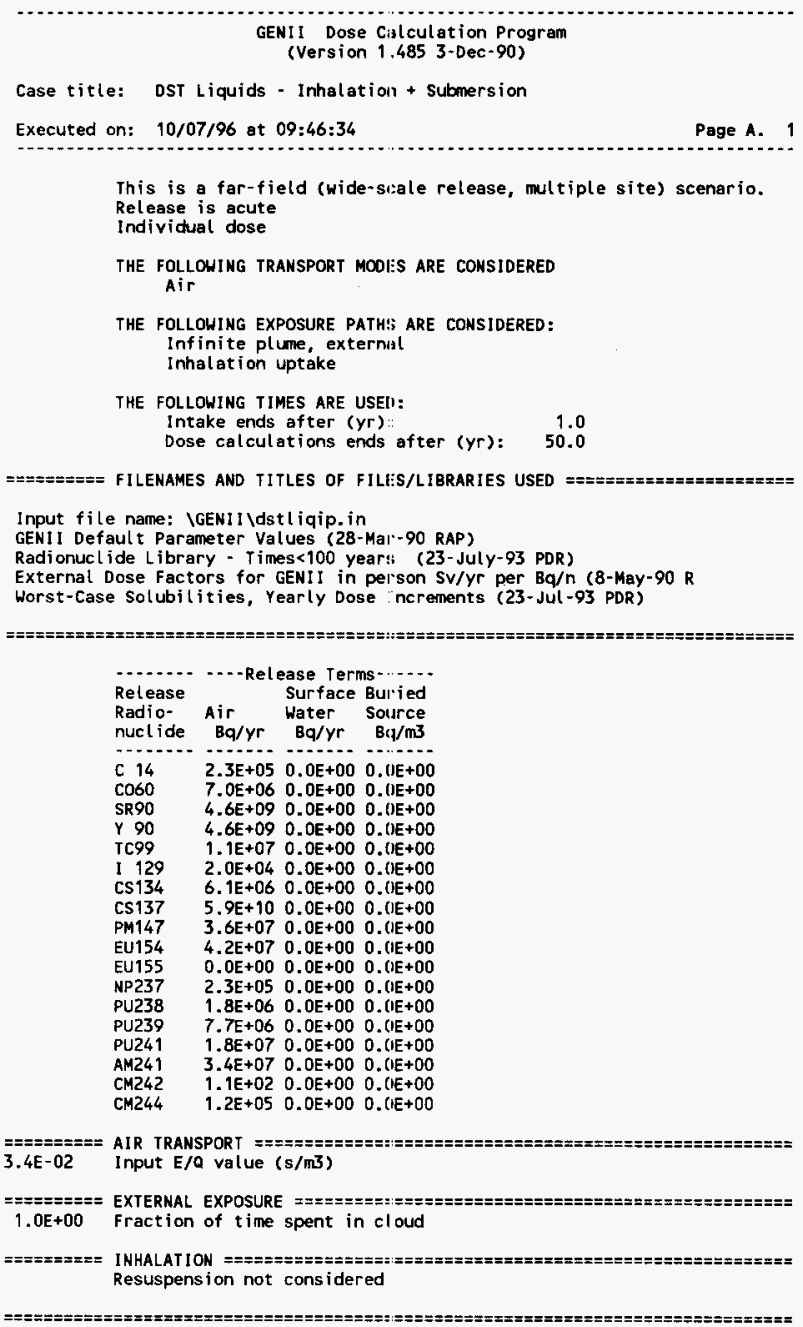




\section{WHC-SD-WM-CN-080, Rev 0}

Input prepared by:

Date:

Input checked by:

Date:

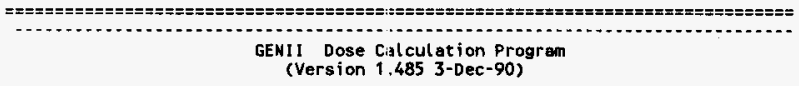

Case title: DST Liquids - Inhalation + Submersion

Executed on: 10/07/96 at 09:46:56

Page C. 1

Acute release

Uptake/exposure period: Dose commitment period: Dose units:

1.0

Sv

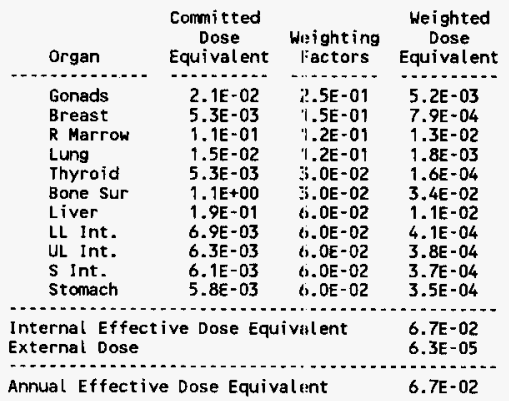

\begin{tabular}{ll} 
Controlling organ: & $\begin{array}{l}\text { Bone Sur } \\
\text { Inh } \\
\text { Controlling Pathway: } \\
\text { Controlling Radionuclide: }\end{array}$ \\
\hline Total Inhalation EDE: & $6.7 \mathrm{~A}-02$ \\
Jotal Ingestion EDE: & $0.0 E+00$
\end{tabular}


WHC-SD-WM-CN-080, Rev 0

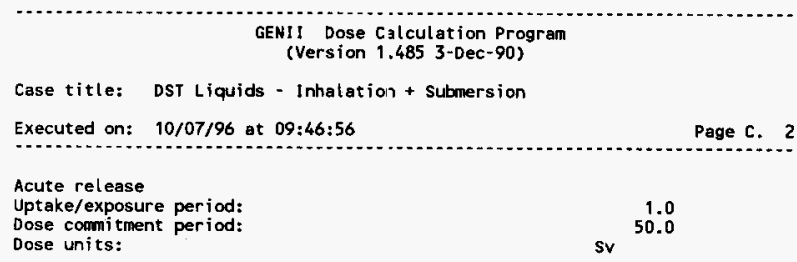

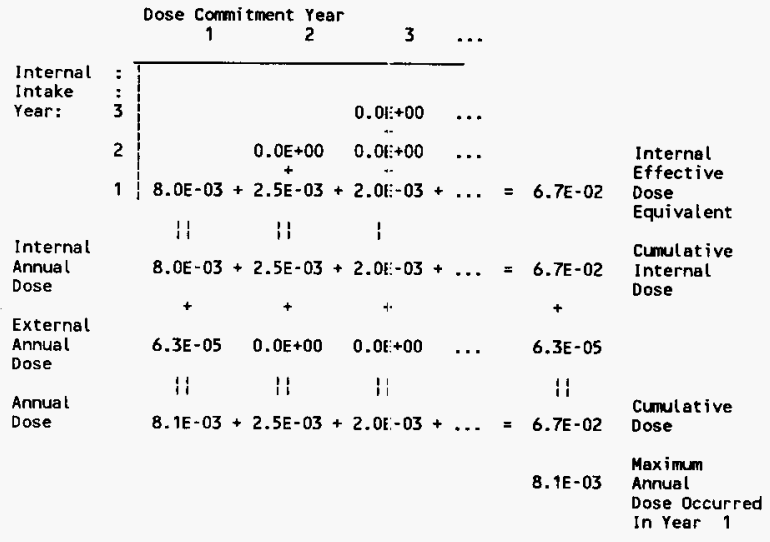


WHC-SD-WM-CN-080, Rev 0

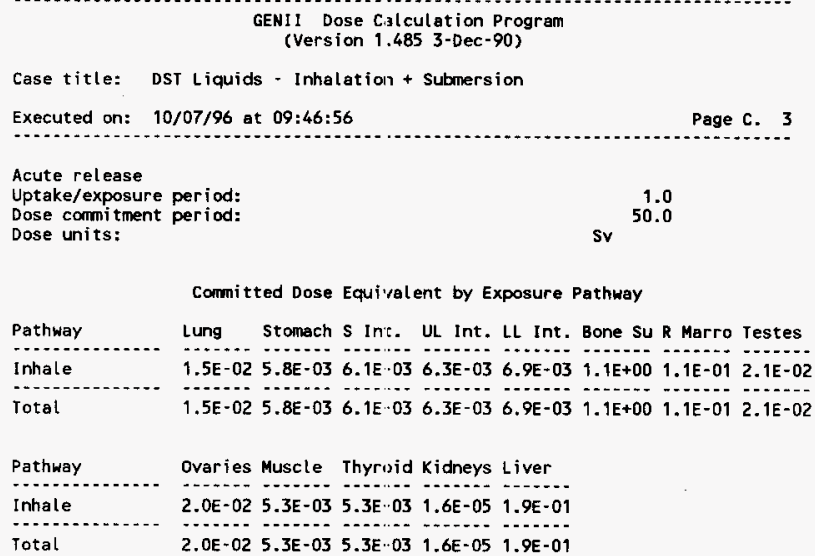

External Dose by Exposure Pathway

Pathway

\begin{tabular}{ll} 
Plume & $6.3 \mathrm{E}-05$ \\
\hline Total & $6.3 \mathrm{E}-05$
\end{tabular}




\section{WHC-SD-WM-CN-080, Rev 0}

GENII Dose Calculation Program

(Version 1.485 3-Dec-90)

Case title: DST Liquids - Inhalatioly + submersion

Executed on: 10/07/96 at 09:46:56

Page C. 4

Acute release

Uptake/exposure period:

Dose commitment period:

Dose units:

1.0

Sv

Comitted Dose Equivalent by Radionucl ide

Radionuclide

c 14

co 60

SR 90

Y 90

TC 99

I 129

CS 134

CS 137

PN 147

EU 154

EU 155

CM 244

CM 242

NP 238

PU 238

PU 241

U 237

AM 241

NP 237

PA 233

PU 239

Total

Lung Stomach $\$$ In'-. UL Int. LL Int. Bone Su R Marro Testes

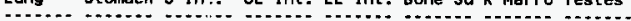
1.5E-09 1.5E-09 1.5E-09 1.5E-09 1.5E-09 1.8E-09 1.8E-09 1.5E-09 2.8E-05 2.2E-06 5.7E-07 7.7E-07 6.3E-07 1.1E-06 1.3E-06 1.3E-07

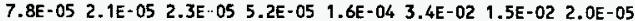
5.0E-04 2.3E-05 5.7E-05 2.8E-04 6.8E-04 8.3E-07 8.3E-07 2.8E-08 2.0E-06 5.5E-07 8.6E-09 2.6E-08 7.0E-08 5.9E-09 5.9E-09 4.9E-09

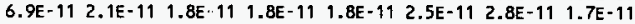
8. IE-07 8.2E-07 9.7E-07 8.9E-07 9.7E-07 7.4E-07 8.2E-07 8.9E-07 5.9E-03 5.7E-03 6.0E-03 5.9E-03 6.0E-03 5.3E-03 5.6E-03 5.8E-03 3.2E-05 1.6E-08 4.0E-08 2.3E-07 6.8E-07 8.3E-06 6.6E-07 4.5E-11 3.7E-05 8.3E-06 7.8E-06 1.1E-05 8.5E-06 2.5E-04 5.0E-05 2.8E-06

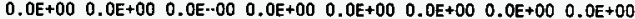

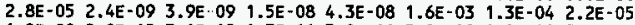

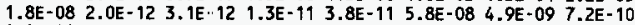

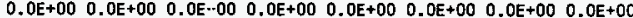
3.6E-04 3.2E-08 5.2E-08 2.2E-07 6.0E-07 3.8E-02 3.1E-03 5.3E-04 $1.5 \mathrm{E}-06 \quad 7.6 \mathrm{E}-09 \quad 8.6 \mathrm{E}-09$ 1.9E-08 $3.2 \mathrm{E}-08$ 8.4E-03 $6.6 \mathrm{E}-04 \quad 1.3 \mathrm{E}-04$

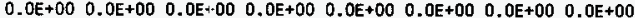
6.9E-03 1.3E-06 $1.7 \mathrm{E}-06 \quad 4.9 \mathrm{E}-06 \quad 1.2 \mathrm{E}-05$ - $8.3 \mathrm{E}-01 \quad 6.5 \mathrm{E}-02 \quad 1.2 \mathrm{E}-02$

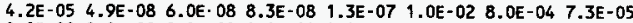

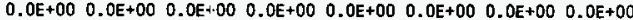
1.5E-03 1.3E-07 2.1E-07 8.4E-07 2.4E-06 1.9E-01 1.5E-02 2.6E-03 ...... 1.5E-02 $5.8 E-03 \quad 6.1 E-03 \quad 6.3 E-03 \quad 6.9 E-03 \quad 1.1 E+00 \quad 1.1 E-01 \quad 2.1 E-02$ 
WHC-SD-WM-CN-080, Rev 0

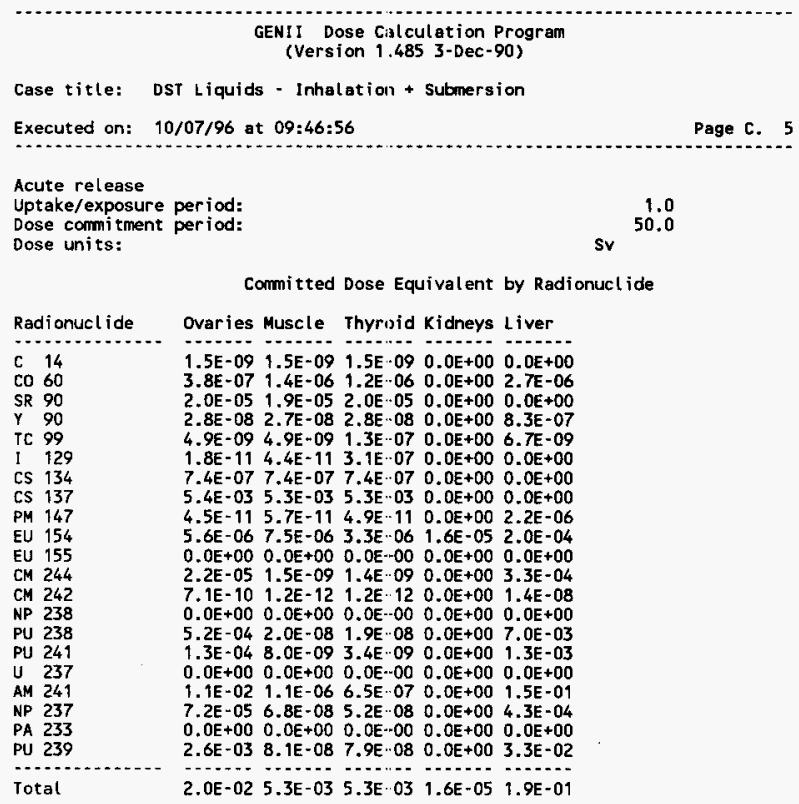


WHC-SD-WM-CN-080, Rev 0

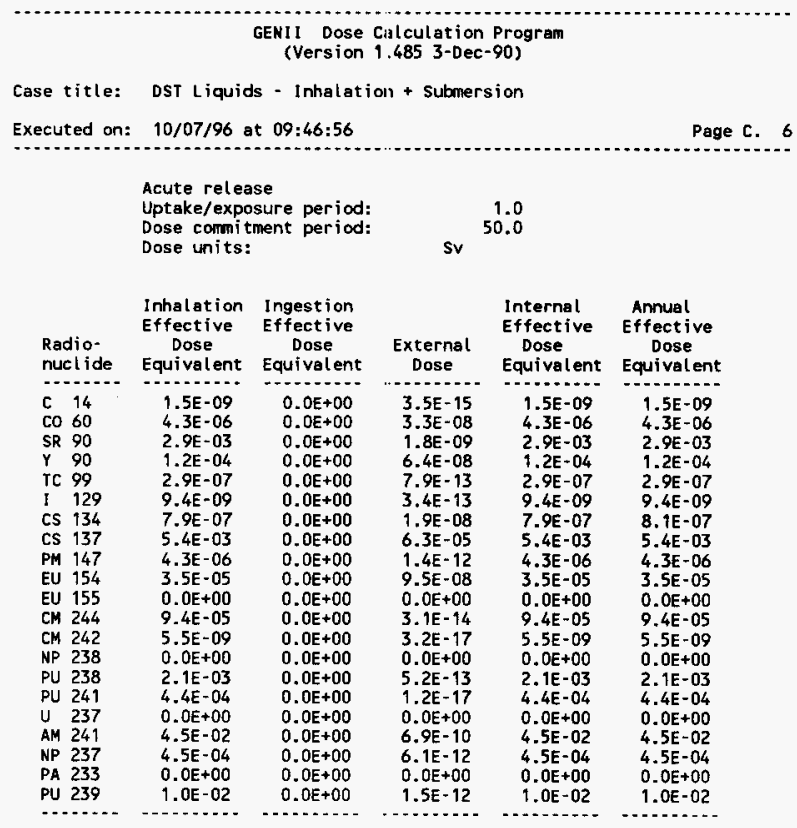




\section{WHC-SD-WM-CN-080, Rev 0}

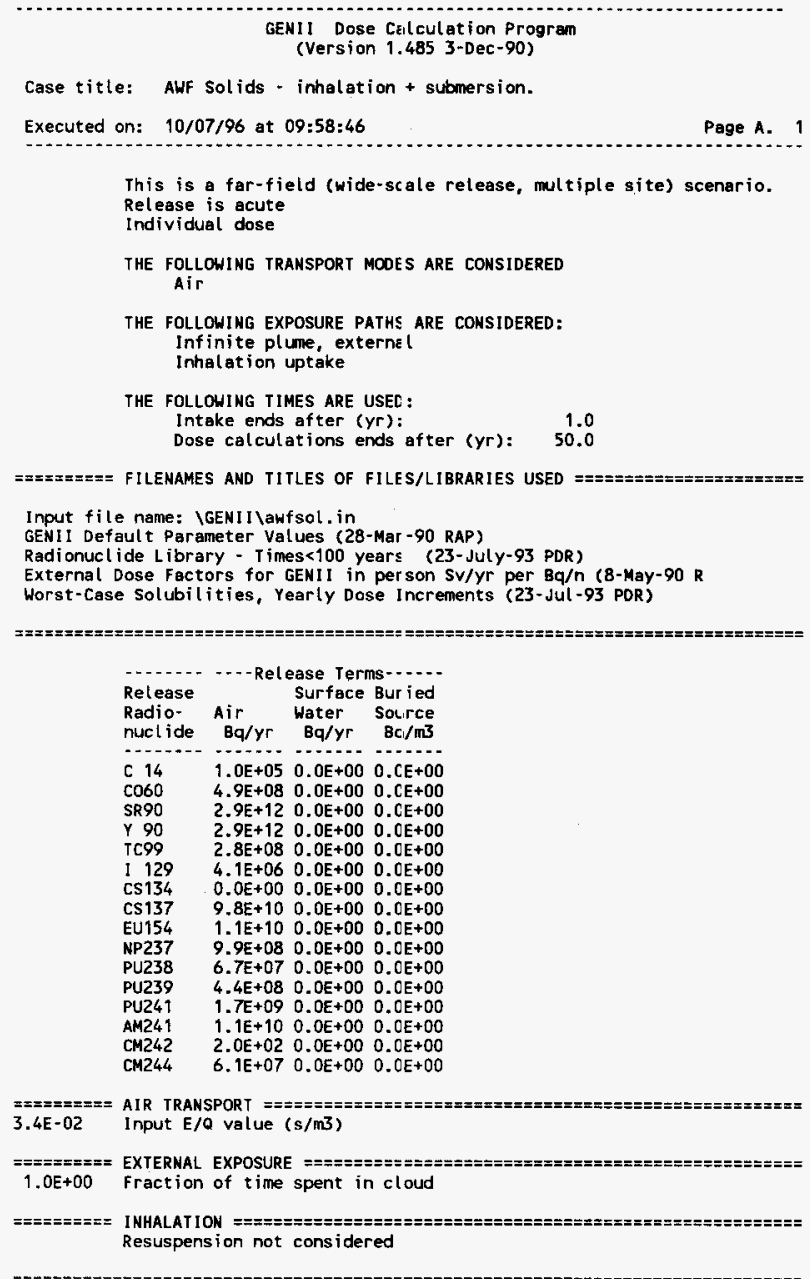


WHC-SD-WM-CN-080, Rev 0

Input checked by:

Date:

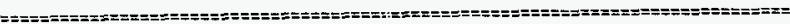




\section{WHC-SD-WM-CN-080, Rev 0}

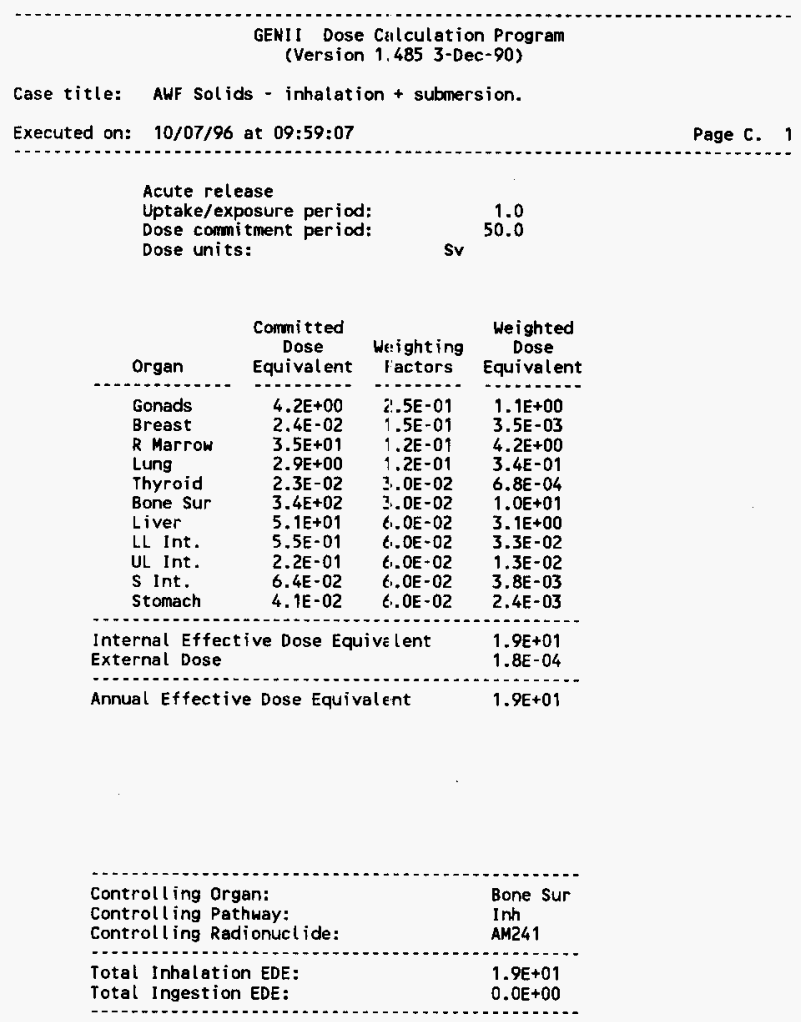




\section{WHC-SD-WM-CN-080, Rev 0}

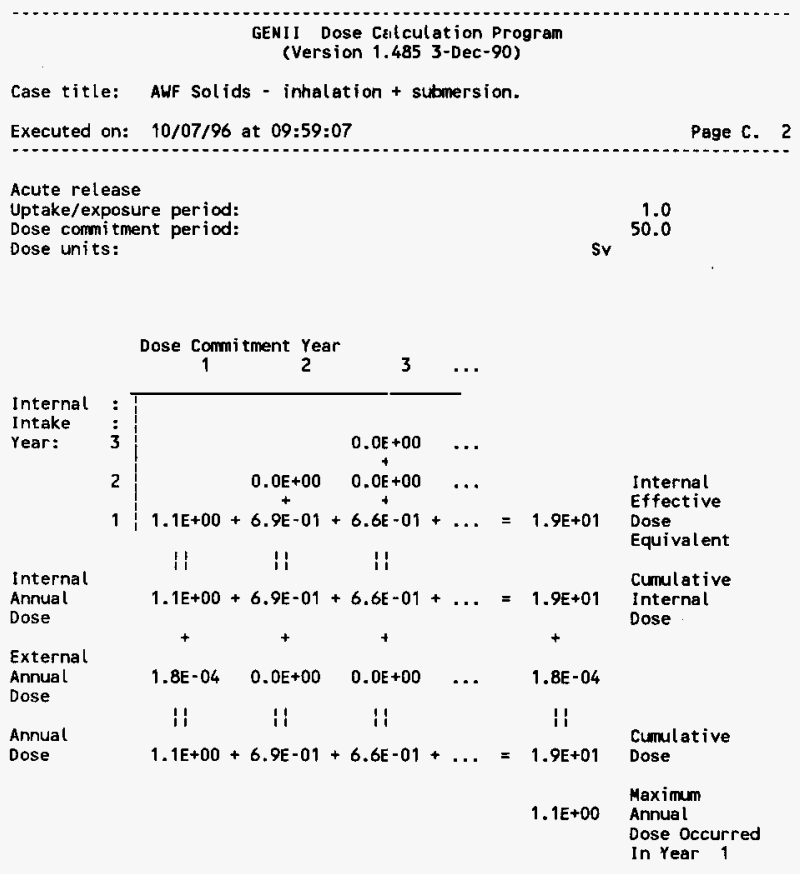




\section{WHC-SD-WM-CN-080, Rev 0}

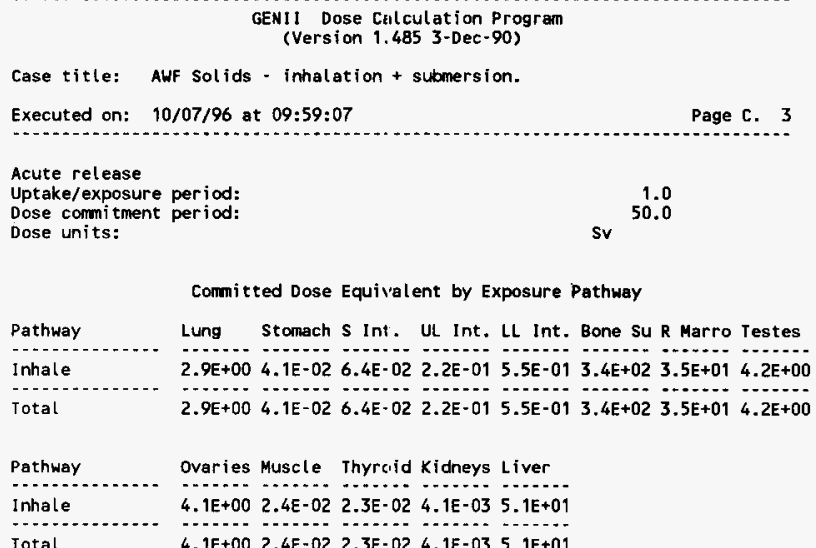


WHC-SD-WM-CN-080, Rev 0

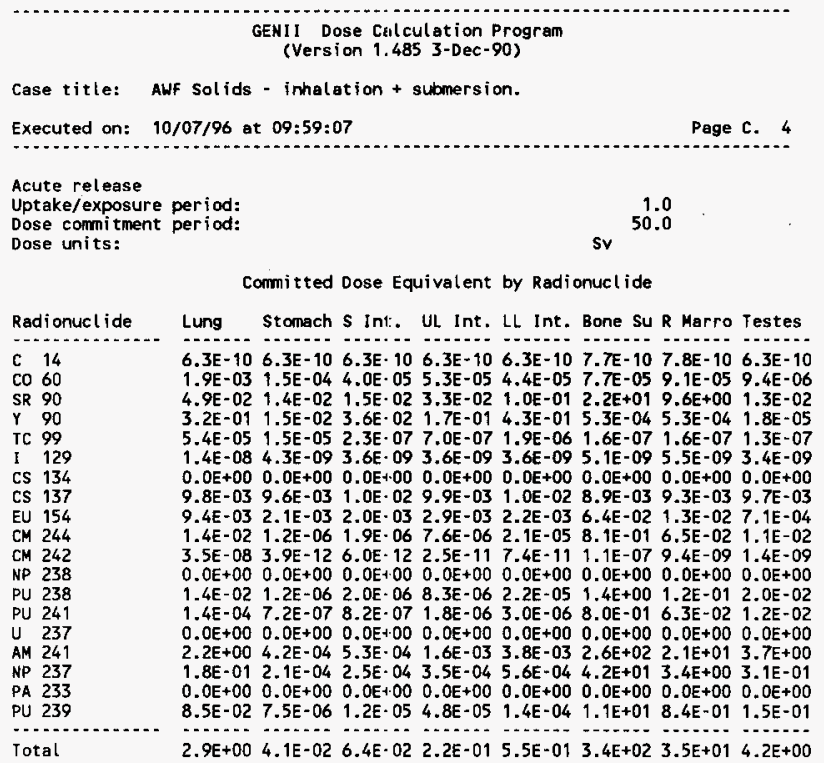




\section{WHC-SD-WM-CN-080, Rev 0}

GEN11 Dose Calcutation Program

(Version 1.485 3-Dec-90)

Case title: AWF solids - inhaletion + submersion.

Executed on: 10/07/96 at 09:59:07

Page C. 5

Acute release

Uptake/exposure period:

Dose commitment period:

Dose units:

1.0

Sv

Committed Dose Equivalent by Radionuclide

Radionucl ide

c 14

CO 60

SR 90

Y 90

TC 99

1129

CS 134

CS 137

EU 154

CM 244

CM 242

NP 238

PU 238

PU 241

U 237

AN 241

NP 237

PA 233

PU 239

Total

Ovaries Muscle Thyroid Kidneys Liver

$6.3 E-10 \quad 6.4 E-10 \quad 6.3 E-10 \quad 0.0 E+000.0 E+00$

2.6E-05 9.9E-05 8.5E-05 $0.0 \mathrm{E}+00 \quad 1.9 \mathrm{E}-04$

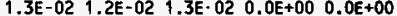

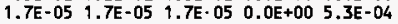

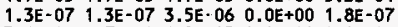

3.6E-09 8.7E-09 6.2E-05 $0.0 \mathrm{E}+00 \quad 0.0 \mathrm{E}+00$

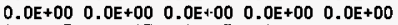

9.0E-03 8.8E-03 8.8E-03 $0.0 E+00 \quad 0.0 E+00$

1.4E-03 1.9E-03 8.5E-04 4.1E-03 5.0E-02

$1.1 \mathrm{E}-02$ 7.6E-07 6.9E-07 $0.0 \mathrm{E}+00$ 1.6E-01

1.4E-09 2.2E- 12 2.2E- $12 \quad 0.0 \mathrm{E}+00$ 2.7E-08

$0.0 E+00 \quad 0.0 E+00 \quad 0.0 E+00 \quad 0.0 E+00 \quad 0.0 E+00$

2. $0 \mathrm{E}-02 \quad 7.5 \mathrm{E}-07 \quad 7.3 \mathrm{E}-07 \quad 0.0 \mathrm{E}+00 \quad 2.6 \mathrm{E}-01$

$1.2 \mathrm{E}-02 \quad 7.6 \mathrm{E}-07$ 3.2E-07 $0.0 \mathrm{E}+00$ 1.2E-01

$\begin{array}{lllll}0.0 E+00 & 0.0 E+00 & 0.0 E+00 & 0.0 E+00 & 0.0 E+00\end{array}$

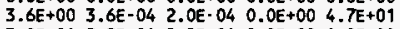

$3.0 E-012.9 E-042.2 E-04 \quad 0.0 E+001.8 E+00$

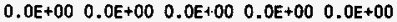

$1.5 \mathrm{E}-01 \quad 4.6 \mathrm{E}-06 \quad 4.6 \mathrm{E}-06 \quad 0.0 \mathrm{E}+00 \quad 1.9 \mathrm{E}+00$

4. $1 E+00 \quad 2.4 E-02 \quad 2.3 E-02 \quad 4.1 E-03 \quad 5.1 E+01$ 


\section{WHC-SD-WM-CN-080, Rev 0}

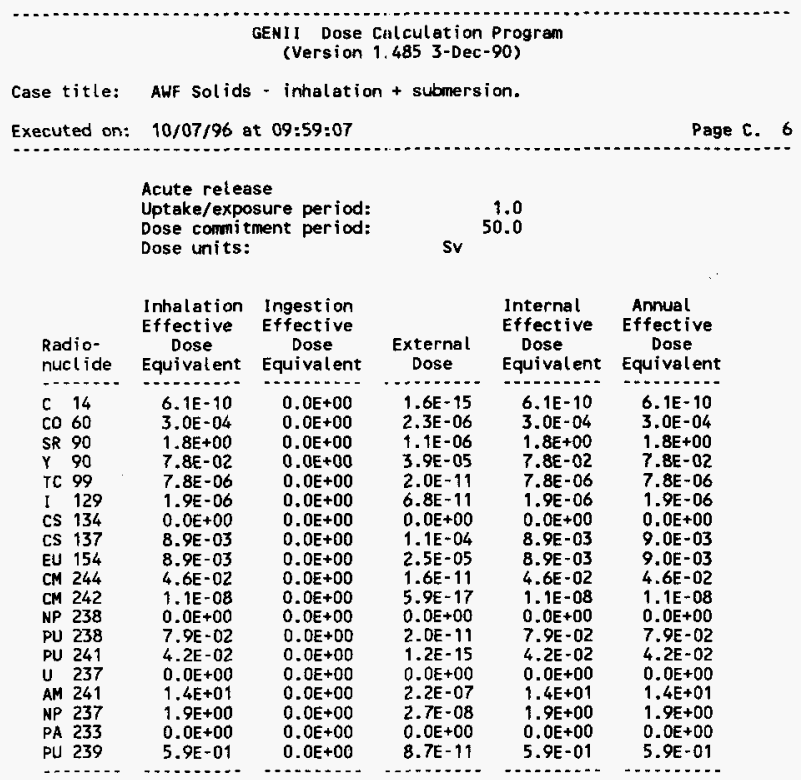




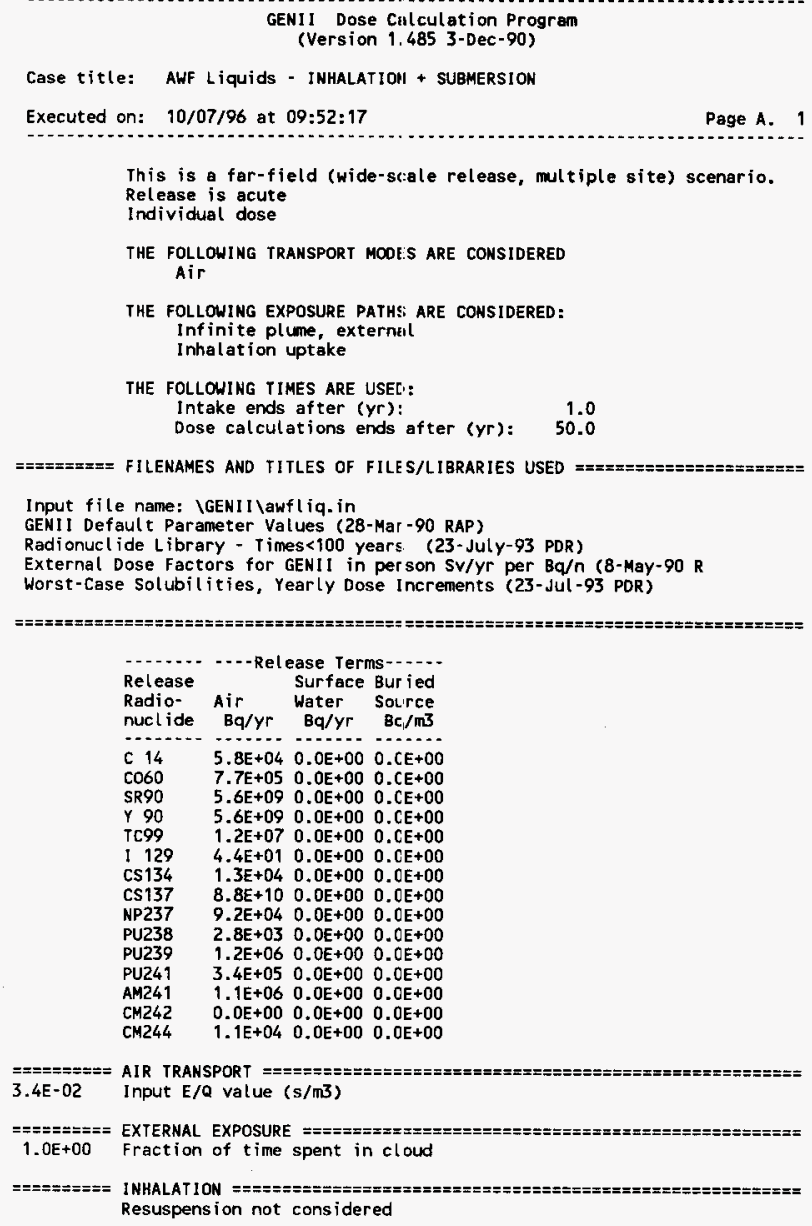

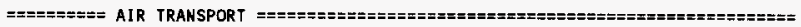
3.4E-02 Input $\mathrm{E} / \mathrm{Q}$ value $(\mathrm{s} / \mathrm{m} 3)$ 
WHC-SD-WM-CN-080, Rev 0

Input checked by:

Date:

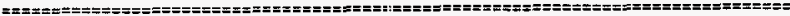


WHC-SD-WM-CN-080, Rev 0

GENII Dose Calculation Program

(Version 1.485 3-Dec-90)

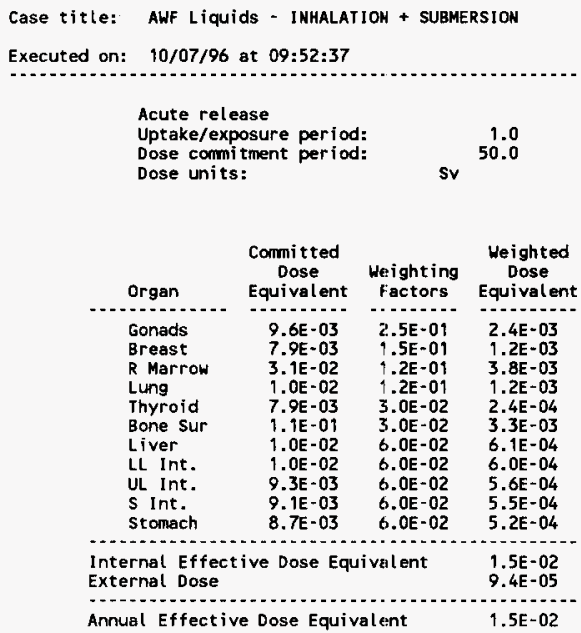

$\begin{array}{ll}\text { Controlling Organ: } & \text { Bone Sur } \\ \text { Controll ing Pathway: } & \text { CS137 } \\ \text { Controll ing Radionuclide: } & 1.5 E-02 \\ \text { Iotal Inhalation EDE: } & 0.0 E+00 \\ \text { Iotal Ingestion EDE: } & \end{array}$


WHC-SD-WM-CN-080, Rev 0

GENII Dose Calculation Program

(Version 1.485 3-Dec-90)

Case title: AWF Liquids - INHALATION + SUBMERSION

Executed on: $10 / 07 / 96$ at $09: 52: 37$

Page C. 2

Acute release

Uptake/exposure period:

Dose commitment period:

Dose units:

1.0

sv

Dose Commi tment Year

1
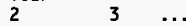

Internal :

Intake

Year:

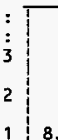

$3 \quad \cdots$

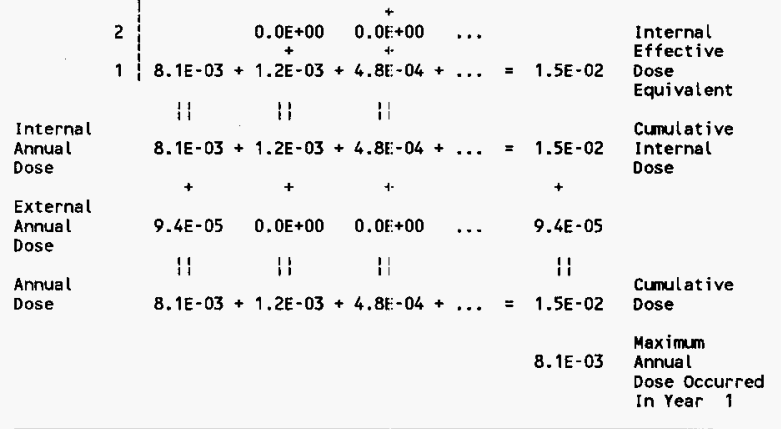




\section{WHC-SD-WM-CN-080, Rev 0}

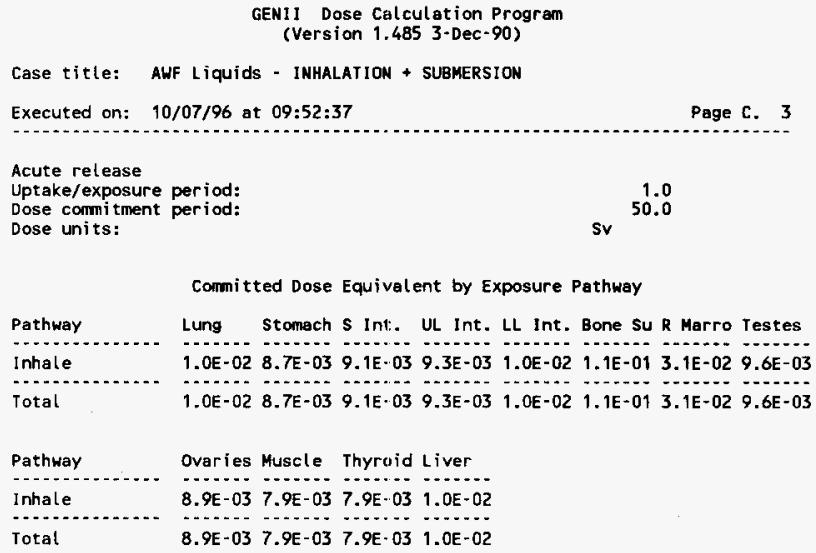




\section{WHC-SD-WM-CN-080, Rev 0}

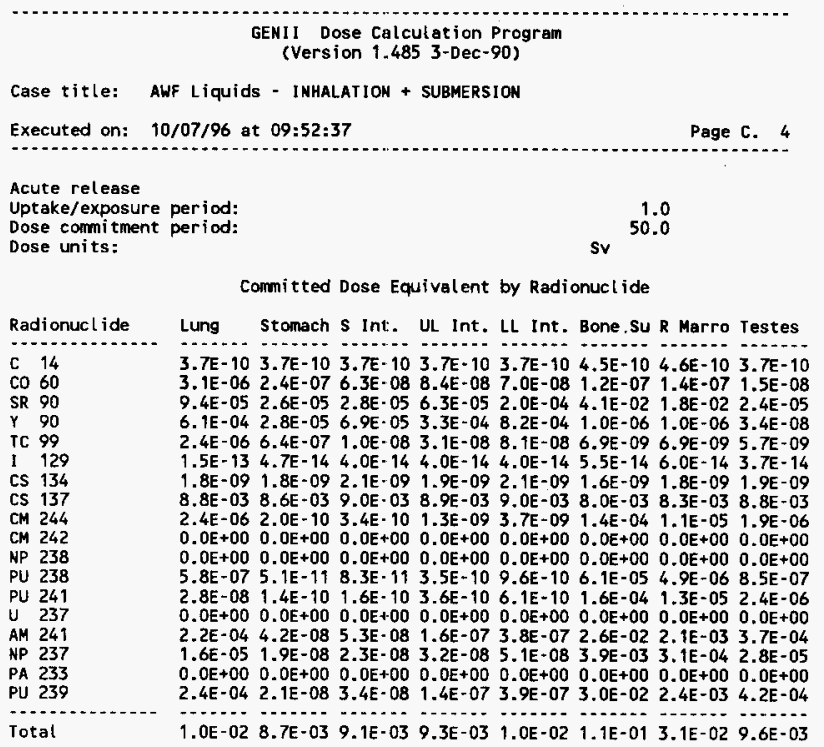




\section{WHC-SD-WM-CN-080, Rev 0}

GENII Dose Calculation Program
(Version 1.485 3-Dec-90)




\section{WHC-SD-WM-CN-080, Rev 0}

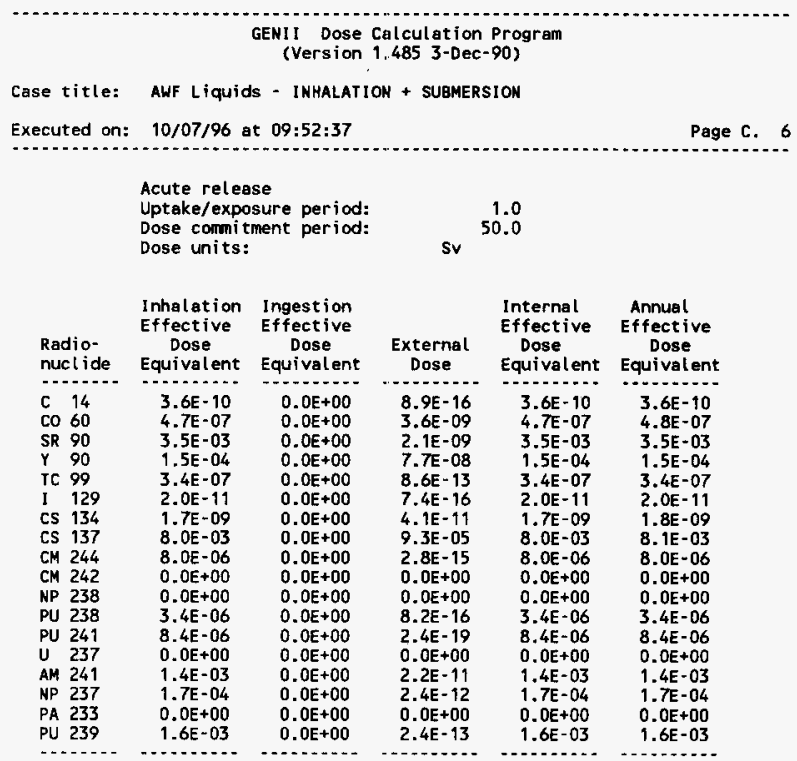


WHC-SD-WM-CN-080, Rev 0

Attachment 2 GXQ Output File 


\section{WHC-SD-WM-CN-080, Rev 0}

Current Input File Name; grnd. IN

GXo Version 4.0

December 19, 1994

General Purpose Atmospheric Dispersion Code

Produced by West inghouse Hanford Company

Users Guide documented in WHC-SO-GN-SWO-30002 Rev. 1.

Val idation documented in HHC-SD-GN-SWD-30003 Rev. 1.

Code Custodian is: Brit E. Hey

Westinghouse Hanford Company

P.o. Box 1970

Richland, WA 99352

(509) $376-2921$

Run Date $=10 / 02 / 96$

Run Time $=10: 45: 07.58$

INPUT ECHO:

TURS FSAR $80,100,120 \mathrm{~m} \mathrm{X/QS}$

c GXo Version 4.0 Input File

c mode

1

c MOOE CHOICE:

$c$ mode $=1$ then $X / a$ based on Hanford site specific meteorology

$c$ mode $=2$ then $X / a$ based on otmospheric stabil $i$ ty class and wind speed

$c$ mode $=3$ then $X / Q$ plot file is created

c LOGICAL CHOICES:

c ifox inorm icdf ichk isite ipop

$T \quad F \quad F \quad F \quad F \quad F$

$c$ ifox $=t$ then joint frequency used to compute frequency to exceed $x / Q$

$c=f$ then joint frequency used to compute annual average $x / 0$

$c$ inorm $=t$ then joint frequency data is normalized (as in GENII)

$=f$ then joint frequency data is un-normal ized

$c$ icdf $=t$ then cumulative distribution $f i$ le created (CDF.OUT)

$=f$ then no cumulative distribution file created

$c$ ichk $=t$ then $X / Q$ parameter print option turned on

$=f$ then no parameter print

$c$ isite $=t$ then $X / Q$ based on joint frequency data for all 16 sectors

$=f$ then $X / Q$ based on joint frequency data of individual sectors

ipop $=t$ then $X / Q$ is population weighted

$=f$ then no population weighting

C $X / Q$ AND HIND SPEED ADJUSTMENT MODELS:

ipuff idep isrc iwind

DIFFUSION COEFFICIENT AD JUSIMENT MODE:LS:

C iwake ipm iflow ientr

EFFECTIVE RELEASE HEIGHT ADJUSTMENT MODELS:

c (irise igrnd) iwash igrav

000000

$c$ ipuff $=1$ then $X / Q$ calculated using puff model

$=0$ then $X / Q$ calculated using defaul $t$ cont inuous plume model

idep $=1$ then plume depletion model turned on (Chamberlain model)

$c$ isre $=1$ then $x / Q$ multiplied by scalar

$=2$ then $X / Q$ adjusted by wind speed function

iwind $=1$ then wind speed corrected for plume height

iwake $=1$ then NRC RG 1.145 building wake model turned on

$=2$ then MACCS virtual distance building wake model turned on

$\mathrm{ipm}=1$ then NRC RG 1.145 pl ume meander model turned on

$=2$ then 5 th Power Law plume meander model turned on

$=3$ then sector average model turned on

c iflow = 1 then sigmas adjusted for volume flow rate

$c$ ientr $=1$ then method of Pasquill used to account for entrainment 


\section{WHC-SD-WM-CN-080, Rev 0}

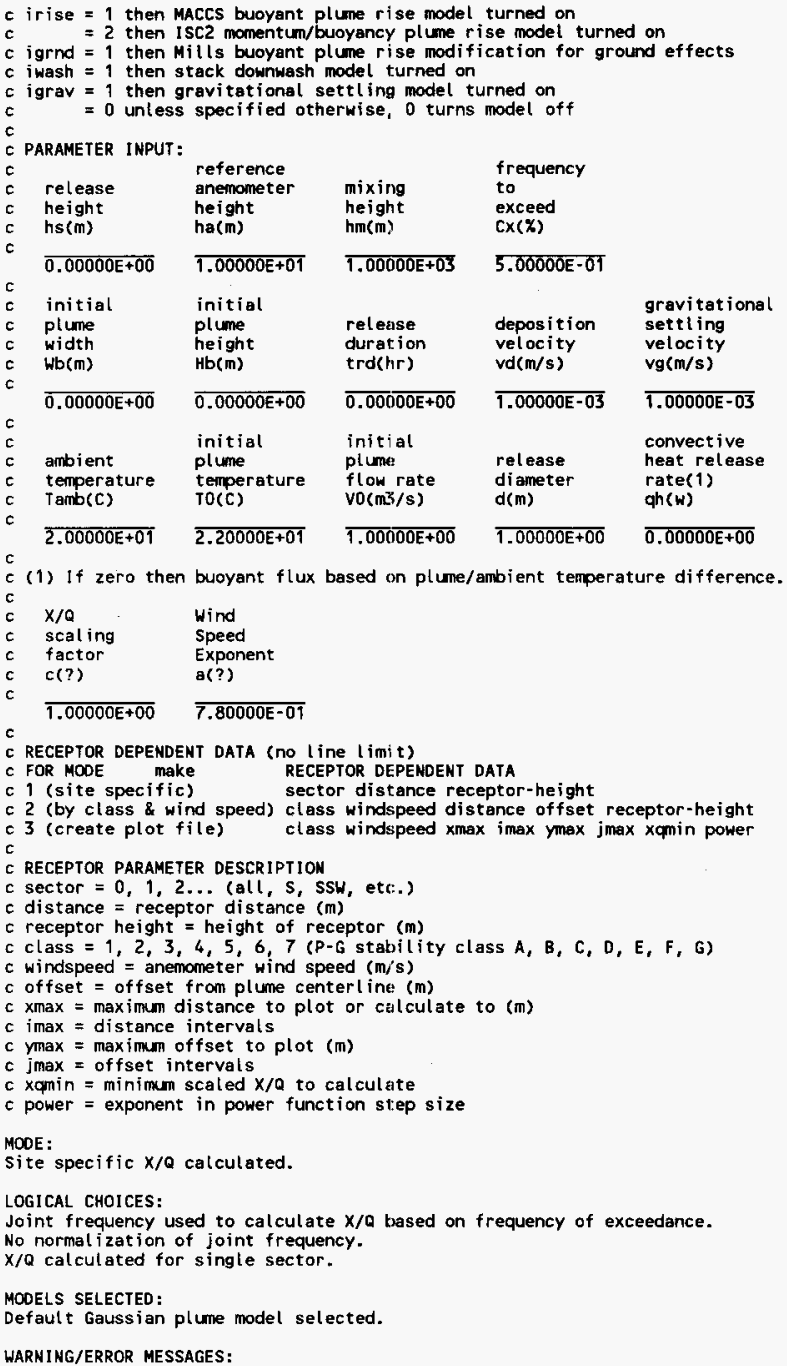




\section{WHC-SD-WM-CN-080, Rev 0}

JOINT FREQUENCY DATA:

200 AREA (HMS) - 10 M - Pasquill A - G (1983 - 1991 Average)

Created $8 / 26 / 92$ KR

TWRS FSAR $80,100,120 \mathrm{~m} \times / Q \mathrm{~S}$

\begin{tabular}{|c|c|c|c|c|c|c|c|c|}
\hline SECTOR & $\begin{array}{l}\text { DISTANCE } \\
\text { (m) }\end{array}$ & $\begin{array}{c}\text { RECEPT } \\
\text { HEIGHT } \\
\text { ( } \mathrm{mi} \text { ) }\end{array}$ & $\begin{array}{l}\text { SECT, } \\
\text { FREQ. } \\
(\chi)\end{array}$ & POPULATION & $\begin{array}{c}\text { TOTAL } \\
\text { POPULATION } \\
\text { SCALED } \\
X / Q \\
(\mathrm{~s} / \mathrm{m} 3)\end{array}$ & $\begin{array}{l}\text { AVERAGE } \\
\text { INDIVIDUAL } \\
\text { SCALED } \\
x / 0 \\
(\mathrm{~s} / \mathrm{m} 3)\end{array}$ & $\begin{array}{l}\text { ATM. } \\
\text { STAB. } \\
\text { CLASS }\end{array}$ & $\begin{array}{l}\text { WIND } \\
\text { SPEED } \\
\text { (m/s) }\end{array}$ \\
\hline $\begin{array}{l}E \\
E \\
E\end{array}$ & $\begin{array}{r}80 \\
90 \\
100 \\
110 \\
120\end{array}$ & $\begin{array}{l}0 \\
0 \\
0 \\
0 \\
0\end{array}$ & $\begin{array}{l}14.05 \\
14.05 \\
14.05 \\
14.05 \\
14.05\end{array}$ & $\begin{array}{l}1 \\
1 \\
1 \\
1 \\
1\end{array}$ & $\begin{array}{l}4.99 \mathrm{E}-02 \\
4.08 \mathrm{E}-02 \\
3.41 \mathrm{E}-02 \\
2.88 \mathrm{E}-02 \\
2.48 \mathrm{E}-02\end{array}$ & $\begin{array}{l}4.99 \mathrm{E}-02 \\
4.08 \mathrm{E}-02 \\
3.41 \mathrm{E}-02 \\
2.88 \mathrm{E}-02 \\
2.48 \mathrm{E}-02\end{array}$ & $\begin{array}{l}\bar{F} \\
F \\
F \\
F \\
F\end{array}$ & $\begin{array}{l}0.89 \\
0.89 \\
0.89 \\
0.89 \\
0.89\end{array}$ \\
\hline
\end{tabular}


WHC-SD-WM-CN-080, Rev 0

Attachment 3 MICROSHIELD Output File 


\section{WHC-SD-WM-CN-080, Rev 0}

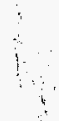

Microshield 3.12

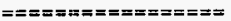
(WESTINGHOUSE HANFORD CO. - \#090)

Page : 1

File : GRNDDEP3.MSH

Run date: October 15, 1996

Run time: $4: 57$ p.m.

File Ref:

Date:

By:

Checked:

CASE: ground deposition $40 \times 11.58 \mathrm{~m} v d=0.01 \mathrm{~m} / \mathrm{s}$

GEOMETRY 11: Rectangular solid source - slab shields

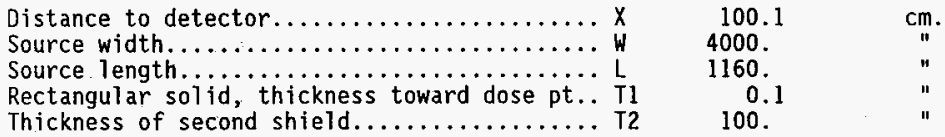

Source Volume: 464000 cubic centimeters

MATERIAL DENSITIES $(\mathrm{g} / \mathrm{cc})$ :

\begin{tabular}{lcc} 
Materiál & Source & Shield 2 \\
\hline Air & & .001220 \\
Aluminum & & \\
Carbon & & \\
Concrete & 1.40 \\
Hydrogen & & \\
Iron & \\
Lead & \\
Lithium & \\
Nickel & \\
Tin & \\
Titanium & \\
Tungsten & \\
Urania \\
Uranium \\
Water \\
Zirconium
\end{tabular}


WHC-SD-WM-CN-080, Rev 0

Page 2

File: GRNDDEP3.MSH

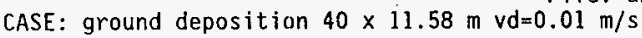

BUILDUP FACTOR: based on TAYLOR method.

Using the characteristics of the materials in shield 1.

\section{INTEGRATION PARAMETERS:}

Number of 1 ateral angle segments (Ntheta)..... 11

Number of azimuthal angle segments (Npsi)..... 11

Number of radial segments (Nradius)........... 11

SOURCE NUCLIDES:

\begin{tabular}{lllllc} 
Nuclide & \multicolumn{1}{c}{ Curies } & Nuclide & \multicolumn{1}{c}{ Curies } & Nuclide & \multicolumn{1}{c}{ Curies } \\
\hline Ba-137m & $1.6027 \mathrm{e}+00$ & Co-60 & $7.1081 \mathrm{e}-03$ & Cs-137 & $1.6946 \mathrm{e}+00$ \\
Eu-154 & $9.8108 \mathrm{e}-02$ & Eu-155 & $8.4595 \mathrm{e}-05$ & &
\end{tabular}

RESULTS:

\begin{tabular}{ccccc}
$\begin{array}{c}\text { Group } \\
\#\end{array}$ & $\begin{array}{c}\text { Energy } \\
\text { (MeV) }\end{array}$ & $\begin{array}{c}\text { Activity } \\
\text { (photons/sec) }\end{array}$ & $\begin{array}{c}\text { Dose point flux } \\
\text { MeV } /(\mathrm{sq} \mathrm{cm}) / \mathrm{sec}\end{array}$ & $\begin{array}{c}\text { Dose rate } \\
(\mathrm{mr} / \mathrm{hr})\end{array}$ \\
\hline 1 & 1.3049 & $1.717 \mathrm{e}+09$ & $-3.333 \mathrm{e}+02$ & $9.676 \mathrm{e}-01$ \\
2 & 1.0401 & $1.312 \mathrm{e}+09$ & $3.270 \mathrm{e}+02$ & $6.260 \mathrm{e}-01$ \\
3 & .7790 & $1.445 \mathrm{e}+09$ & $2.726 \mathrm{e}+02$ & $5.510 \mathrm{e}-01$ \\
4 & .6638 & $5.365 \mathrm{e}+10$ & $8.745 \mathrm{e}+03$ & $1.814 \mathrm{e}+01$ \\
5 & .4766 & $7.860 \mathrm{e}+06$ & $9.460 \mathrm{e}-01$ & $1.930 \mathrm{e}-03$ \\
6 & .4453 & $1.830 \mathrm{e}+07$ & $2.061 \mathrm{e}+00$ & $4.215 \mathrm{e}-03$ \\
7 & .3984 & $7.602 \mathrm{e}+06$ & $7.680 \mathrm{e}-01$ & $1.576 \mathrm{e}-03$ \\
8 & .2422 & $2.397 \mathrm{e}+08$ & $1.488 \mathrm{e}+01$ & $2.890 \mathrm{e}-02$ \\
9 & .1953 & $8.246 \mathrm{e}+06$ & $4.147 \mathrm{e}-01$ & $7.601 \mathrm{e}-04$ \\
10 & .1172 & $1.469 \mathrm{e}+09$ & $4.489 \mathrm{e}+01$ & $7.085 \mathrm{e}-02$ \\
11 & & & & \\
12 & & & & \\
13 & & & & \\
14 & & & & \\
15 & & & & \\
16 & & & & \\
17 & & & & \\
18 & & & & \\
19 & & & &
\end{tabular}


WHC-SD-WM-CN-080, Rev 0

Attachment 4 Peer Review and HEDOP Checklists 


\section{WHC-SD-WM-CN-080, Rev 0}

\section{PEER REVIEW CHECKLIST}

Document Reviewed: Comparison of Radiological Dose Pathways for Tank Farm Accidents, WHC-SD-WM-CN-80

Author: J. C. Van Keuren

Date: October 1996

Scope of Review: Entire Document

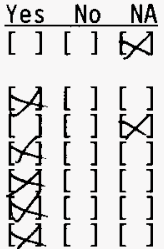

[ ] [ ] $\infty$

$\infty[$ ] [ ]

$\infty[$ ] [ ]

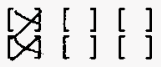

[] [ ] $[x]$

$\left.\begin{array}{llll}{[} & {[} & \infty & \infty \\ 凶 & {[} & 0 & {[}\end{array}\right]$

[ ] [ ] $\bowtie$

[ ] [ ] $\bigotimes$

Previous reviews complete and cover analysis, up to scope of this review, with no gaps.

Problem completely defined.

Accident scenarios developed in a clear and logical manner.

Necessary assumptions explicitly stated and supported.

Computer codes and data files documented.

Data used in calculations explicitly stated in document.

Data checked for consistency with original source information as applicable.

Mathematical derivations checked including dimensional consistency of results.

Models appropriate and used within range of validity or use outside range of established validity justified.

Hand calculations checked for errors. Spreadsheet results should be treated exactly the same as hand calculations.

Software input correct and consistent with document reviewed.

Software output consistent with input and with results reported in document reviewed.

Limits/criteria/guidelines applied to analysis results are appropriate and referenced. Limits/criteria/guidelines checked against references. Safety margins consistent with good engineering practices.

Conclusions consistent with analytical results and applicable limits. Results and conclusions address all points required in the problem statement.

Format consistent with appropriate NRC Regulatory Guide or other standards

Review calculations, comments, and/or notes are attached.

\section{D. [ ] [ ] Document approved.}

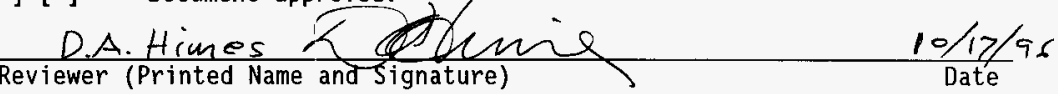




\author{
WHC-SD-WM-CN-080, Rev 0
}

\title{
HEDOP REVIEW CHECKLIST
}

Document Reviewed: Comparison of Radiological Dose Pathways for Tank Farm Accidents, WHC-SD-WM-CN-80

Author: J. C. Van Keuren

Date: $\quad$ October 1996

Scope of Review: Entire Document

YES NO* N/A

B] [ ] [ ] 1. A detailed technical review and approval of the environmenta] transport and dose calculation portion of the analysis has been performed and documented.

[ ] [ ]

2. Detailed technical review(s) and approval(s) of scenario and release determinations have been performed and documented.

$\left[\begin{array}{lll}] & {[}\end{array}\right]$

3. HEDOP-approved code(s) were used.

4. Receptor locations were selected according to HEDOP recommendations.

[ ] [ ] D

$\left[\begin{array}{lll}3 & {[} & {[}\end{array}\right]$

5. All applicable environmental pathways and code options were included and are appropriate for the calculations.

6. Hanford site data were used.

7. Model adjustments external to the computer program were justified and performed correctly.

$\left[\begin{array}{lll}3 & {[}\end{array}\right]$

8. The analysis is consistent with HEDOP recommendations.

9. Supporting notes, calculations, comments, comment resolutions, or other information is attached. (Use the "Page 1 of $X "$ page numbering format and sign and date each added page.)

[ ] ]

10. Approval is granted on behalf of the Hanford Environmental Dose Overview Panel.

* All "NO" responses must be explained and use of nonstandard methods justified.

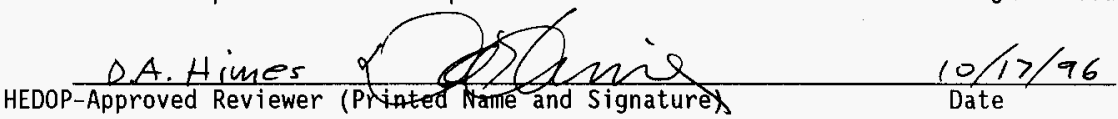

CONMENTS (add additional signed and dated pages if necessary): 


\section{DISTRIBUTION SHEET}

\begin{tabular}{|c|c|c|c|c|c|}
\hline \multirow{2}{*}{$\begin{array}{l}\text { To } \\
\text { Distribution }\end{array}$} & \multirow{2}{*}{\multicolumn{3}{|c|}{$\begin{array}{l}\text { From } \\
\text { Consequence Analysis }\end{array}$}} & \multicolumn{2}{|l|}{ Page 1 of 1} \\
\hline & & & & \multicolumn{2}{|c|}{ Date October 17, 1996} \\
\hline \multirow{2}{*}{\multicolumn{4}{|c|}{$\begin{array}{l}\text { Project TitleMork Order } \\
\text { Comparison of Radiological Dose Pathways for Tank Farm Accidents }\end{array}$}} & \multicolumn{2}{|c|}{ EDT No. 619416} \\
\hline & & & & \multicolumn{2}{|l|}{ ECN No. } \\
\hline \multicolumn{2}{|l|}{ Name } & $\begin{array}{c}\text { Text } \\
\text { With All } \\
\text { Attach. }\end{array}$ & Text Only & $\begin{array}{l}\text { Attach./ } \\
\text { Appendix } \\
\text { Only }\end{array}$ & $\begin{array}{l}\text { EDT/ECN } \\
\text { Only }\end{array}$ \\
\hline $\begin{array}{l}\text { C. Carro } \\
\text { G. L. Dunford } \\
\text { D. A. Himes } \\
\text { D. S. Leach } \\
\text { J. C. Van Keuren } \\
\text { TWRS S\&L Project Files } \\
\text { Central Files }\end{array}$ & $\begin{array}{l}\text { A2-34 } \\
\text { A2-34 } \\
A 3-34 \\
A 3-34 \\
A 3-34 \\
A 2-26 \\
A 3-88\end{array}$ & $\begin{array}{l}X \\
X \\
X \\
X \\
X \\
X \\
X\end{array}$ & & & \\
\hline
\end{tabular}

